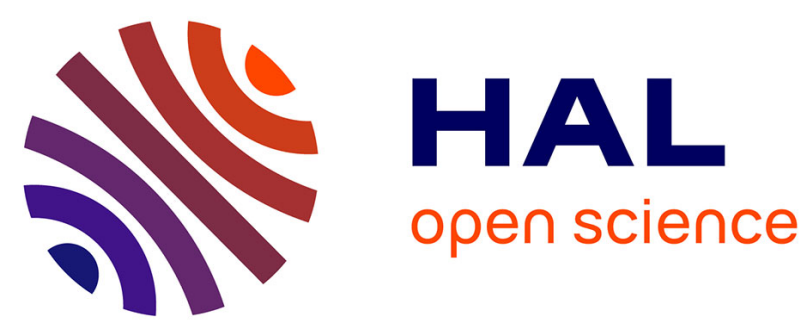

\title{
ALMOST AUTOMORPHIC SOLUTIONS FOR NONAUTONOMOUS PARABOLIC EVOLUTION EQUATIONS
}

Mahmoud Baroun, Khalil Ezzinbi, Kamal Khalil, Lahcen Maniar

\section{> To cite this version:}

Mahmoud Baroun, Khalil Ezzinbi, Kamal Khalil, Lahcen Maniar. ALMOST AUTOMORPHIC SOLUTIONS FOR NONAUTONOMOUS PARABOLIC EVOLUTION EQUATIONS. Semigroup Forum, In press. hal-02019534

\section{HAL Id: hal-02019534 \\ https://hal.science/hal-02019534}

Submitted on 14 Feb 2019

HAL is a multi-disciplinary open access archive for the deposit and dissemination of scientific research documents, whether they are published or not. The documents may come from teaching and research institutions in France or abroad, or from public or private research centers.
L'archive ouverte pluridisciplinaire HAL, est destinée au dépôt et à la diffusion de documents scientifiques de niveau recherche, publiés ou non, émanant des établissements d'enseignement et de recherche français ou étrangers, des laboratoires publics ou privés. 


\title{
Almost AUTOMORPHIC SOLUTIONS FOR NONAUTONOMOUS PARABOLIC EVOLUTION EQUATIONS
}

January 29, 2019

\author{
Mahmoud Baroun $^{1}$, Khalil Ezzinbi ${ }^{1}$, Kamal Khali1 ${ }^{1}$ and Lahcen Maniar ${ }^{1}$ \\ ${ }^{1}$ Cadi Ayyad University, Faculty of Sciences Semlalia, Departement of Mathematics, Marrakesh B.P. 2390-40000, Mo- \\ rocco.
}

Abstract. In this work, we study the existence and uniqueness of an almost automorphic solution to semilinear nonautonomous parabolic evolution equations with inhomogeneous boundary conditions using the exponential dichotomy. We assume that the homogeneous problem satisfies the "Acquistapace-Terreni" conditions and that the forcing terms are Stepanov-like almost automorphic. An example is given for illustration.

Keywords. Parabolic evolution equations, Stepanov almost automorphic functions, Evolution family, Extrapolation and interpolation spaces, exponential dichotomy, $\mu$-pseudo almost automorphic functions.

\section{Introduction}

In this work, we study the existence and uniqueness of a $\mu$-pseudo almost automorphic mild solutions of the following semilinear parabolic evolution equation with inhomogeneous boundary conditions:

$$
\left\{\begin{aligned}
u^{\prime}(t) & =A_{m}(t) u(t)+f(t, u(t)), t \in \mathbb{R} \\
B(t) u(t) & =g(t, u(t)), t \in \mathbb{R} .
\end{aligned}\right.
$$

Where $\left(A_{m}(t), D\left(A_{m}(t)\right)\right), t \in \mathbb{R}$ is a family of linear operators defined in a Banach space $X$, for $t \in \mathbb{R}$, $B(t): D\left(A_{m}(t)\right) \longrightarrow \partial X$ is a boundary linear operator, where $\partial X$ is a boundary space (see Section 6 for an example of such spaces). It is assumed that, $A_{m}(\cdot), B(\cdot)$ are Stepanov-like almost automorphic and that $A(t) t \in \mathbb{R}$ satisfy the "Acquistapace-Terreni" conditions and has an exponential dichotomy on $\mathbb{R}$, where $A(t):=A_{m}(t) \mid \operatorname{ker}(B(t)), t \in \mathbb{R}$. The functions $f: \mathbb{R} \times X_{\beta}^{t} \longrightarrow X$ and $g: \mathbb{R} \times X_{\beta}^{t} \longrightarrow \partial X$ are Stepanovlike $\mu$-pseudo almost automorphic, where $X_{\beta^{\prime}}^{t} 0<\beta<1$ are some continuous interpolation spaces with respect to the linear operators $A(t), t \in \mathbb{R}$.

The concept of almost automorphy was introduced by S. Bochner [7] as a generalisation of the well known almost perdioc functions. Due to its applications in differential equations, almost automorphic functions have undergone several interesting generalizations in different settings. In [26], J. M. N'Guerekata and A. A. Pankov introduced a new concept of almost automorphy defined on average of measurable functions which are locally integrable, namely, Stepanov-like almost automorphy. Another generalization, this time is in term of perturbation, the so-called pseudo almost automorphy due to J. Liang et al. in [31]. A pseudo almost automorphic function is a perturbation of an almost automorphic fonction by an ergodic term (see Section 2). In [13], T. Diagana gave a generalisation of the concept of pseudo almost automorphic functions by introducing a more general definition of the ergodic perturbation of Zhang [32], namely, the weighted pseudo almsot automorphic functions. After that, in [11], K. Ezzinbi et al. introduced a new concept of pseudo almost automorphic functions called $\mu$-pseudo almost automorphic functions. More precisely, a $\mu$-pseudo almost automorphic function is a perturbation of an almost automorphic function by an ergodic term as in the two previous concepts, but here the authors gave a more general definition of the 
ergodicity in the mean of positive measures in which the other previous definitions are just simple particular cases. Finally, by combining the previous notions we obtain a more general concept of Stepanov-like $\mu$-pseudo almost automorphy introduced by B. Es-sebbar and K. Ezzinbi in [3].

In the litterature, we found several works. In [33], the authors established the existence of weighted pseudo almost automorphic a mild solution to equation (1.1) in the particular case where $g \equiv 0$. Under assumptions that $(A(t))_{t \in \mathbb{R}}$ satisfy "Aquistapace-Terreni" conditions (2.1), (2.2) on a Banach space $X$, the evolution family $(U(t, s))_{t \geq s}$ generated by $(A(t))_{t \in \mathbb{R}}$ has an exponential dichotomy on $\mathbb{R}$. The Green function $\Gamma(\cdot, \cdot)$ is bi-almost automorphic and the function $f$ is Stepanov-like weighted pseudo almost automorphic. In [19], the authors established the existence of pseudo almost automorphic a mild solution to equation (1.1) in the particular case where $g \equiv 0$. Under assumptions that $(A(t))_{t \in \mathbb{R}}$ generates an evolution family $(U(t, s))_{t \geq s}$ which has an exponential dichotomy on $\mathbb{R}$. The Green function $\Gamma(\cdot, \cdot)$ is bi-almost automorphic and the function $f$ is Stepanov-like pseudo almost automorphic.

In this work, we use the approach developed in [5]. We prove the results for the following equivalent evolution equation associated to equation (1.1):

$$
u^{\prime}(t)=A_{\alpha-1}(t) u(t)+\underbrace{f(t, u(t))+\left(\omega-A_{\alpha-1}(t)\right) D(t) g(t, u(t))}_{\tilde{f}(t, u(t))} \quad \text { for all } t \in \mathbb{R},
$$

in the continuous extrapolation spaces $X_{\alpha-1}^{t}$, associated to the sectorial operators $A_{\alpha-1}(t), t \in \mathbb{R}, 0<$ $\alpha<1$, where, $D(t):=\left(B(t) \mid \operatorname{ker}\left(\omega-A_{m}(t)\right)\right)^{-1}, t \in \mathbb{R}$ are the Dirichlet maps for $\omega$ large enough.

We begin by introducing the fact that, if $R(\omega, A(\cdot)) \in A A S^{p}(\mathbb{R}, \mathcal{L}(X))$ it holds that $\Gamma(\cdot, \cdot) \in b A A(\mathbb{R}, \mathcal{L}(X))$, see Theorem 4.1, this is a generalisation to the result introduced in [10] where the authors proved that $\Gamma(\cdot, \cdot) \in b A A(\mathbb{R}, \mathcal{L}(X))$ if $R(\omega, A(\cdot)) \in A A(\mathbb{R}, \mathcal{L}(X))$ which is strong as hypothesis than ours. Therefore, we prove the existence and uniqueness of a $\mu$-pseudo almost automorphic mild solution to the following linear evolution equation:

$$
u^{\prime}(t)=A_{\alpha-1}(t) u(t)+h(t) \quad \text { for all } t \in \mathbb{R},
$$

in the spaces $X_{\alpha-1}^{t}$, where $h$ is Stepanov-like $\mu$-pseudo almost automorphic, see Theorems 4.2 and 4.3 . Using contraction mapping Theorem, we prove the existence of a unique $\mu$-pseudo almost automorphic mild solution to the following semilinear evolution equation:

$$
u^{\prime}(t)=A_{\alpha-1}(t) u(t)+\tilde{f}(t, u(t)) \quad \text { for all } t \in \mathbb{R},
$$

in the spaces $X_{\alpha-1}^{t}$, under the assumption that $\tilde{f}$ is Stepanov-like $\mu$-pseudo almost automorphic and Lipschitzian with respect to the second argument, see Theorem 4.4. Finally, under Greiner's assumptions [20], we prove the result to equation (1.1), see Theorem 5.1.

The organization of this paper is as follows, in Section 2, we recall some results in the interpolation and the extrapolation theory of a family of linear operators, then we introduce some dichotomy estimates in the extrapolated spaces $X_{\alpha-1}$, which are a key fact to prove our main results. After that, we give important properties of $\mu$-pseudo almost automorphic functions in Bochner and Stepanov senses respectively. Section 3 is devoted to a new composition result of $\mu$-pseudo almost automorphic functions in Stepanov sense. In Section 4 , we prove the existence and uniqueness of a $\mu$-pseudo almost automorphic mild solution to equation (1.4), under assumptions that $\tilde{f}$ is $\mu$-pseudo almost automorphic in Stepanov sense and $R(\omega, A(\cdot))$ is Stepanov-like almost automorphic. In Section 5, we show the existence of a unique $\mu$-pseudo almost automorphic mild solution to equation (1.1) under Greiner's assumptions and that $A_{m}(\cdot), B(\cdot)$ are Stepanovlike almost automorphic and the functions $f$ and $g$ are $\mu$-pseudo almost automorphic in Stepanov sense. In Section 6, for illustration, we provide an application for some diffusion problems.

\section{Preliminaries}

In this section, we recall some definitions and notations as we introduce some results needed in the following. Throughout this work, $(X,\|\cdot\|)$ and $\left(Y,\|\cdot\|_{Y}\right)$ are two Banach spaces. $B C(\mathbb{R}, X)$ equipped with the supremum norm, the Banach space of bounded continuous functions $f$ from $\mathbb{R}$ into $X$. Moreover, for 
$1 \leq p<\infty, q$ denotes its conjugate exponent defined by $\frac{1}{p}+\frac{1}{q}=1$ if $p \neq 1$ and $q=\infty$ if $p=1$. By $L_{l o c}^{p}(\mathbb{R}, X)$ (resp. $L^{p}(\mathbb{R}, X)$ ), we designate the space (resp. the Banach space) of all equivalence classes of measurable functions $f$ from $\mathbb{R}$ into $X$ such that $\|f(\cdot)\|^{p}$ is locally integrable (resp. integrable). We denote by $\mathcal{B}(\mathbb{R})$, the Lebesgue $\sigma$-field of $\mathbb{R}$ and by $\mathcal{M}$ the set of all positive measures $\mu$ on $\mathcal{B}(\mathbb{R})$ satisfying $\mu(\mathbb{R})=+\infty$ and $\mu([a, b])<+\infty$, for all $a, b \in \mathbb{R}$ with $a \leq b$.

\subsection{Evolution families and intermediate spaces}

We provide results on interpolation and extrapolation spaces for $A(t), t \in \mathbb{R}$. For more details, we refer to $[2,18,23]$.

Let $(A(t), D(A(t))), t \in \mathbb{R}$ be a family of linear closed operators on a Banach space $X$ that satisfies the conditions introduced by P. Acquistapace and B. Terrini in [1], i.e., there exist constants $\omega \in \mathbb{R}, \theta \in\left(\frac{\pi}{2}, \pi\right), M>$ 0 and $\eta, v \in(0,1]$ with $\eta+v>1$ such that

$$
\begin{aligned}
& \|\lambda R(\lambda, A(t)-\omega)\|_{\mathcal{L}(X)} \leq L, \\
& \|(A(t)-\omega) R(\lambda, A(t)-\omega)[R(\omega, A(t))-R(\omega, A(s))]\|_{\mathcal{L}(X)} \leq \frac{M|t-s|^{\eta}}{|\lambda|^{v}}
\end{aligned}
$$

for all $t \geq s, t, s \in \mathbb{R}$ and $\lambda \in \Sigma_{\omega, \theta}:=\{z \in \mathbb{C}: z \neq 0,|\arg (z)| \leq \theta\} \subset \rho(A(t)-\omega)$. The domains $D(A(t))$ of the operators $A(t)$ may change with $t$ and not required to be dense in $X$.

By the condition (2.1), each operator $A(t)$ generates a bounded analytic semigroup $\left(T_{t}(s)\right)_{s \geq 0}$ where the domains $D(A(t))$ may change with respect to $t$ and not dense in $X$, so that the semigroups may be not strongly continuous at 0 . The condition (2.2) provides some regularity in the dependence on $t$ of the operators $A(t)$. Hence, the conditions (2.1) and (2.2) implies that the operators $A(t)$ generates an evolution family $U(t, s)$ for $t, s \in \mathbb{R}$ with $t \geq s$. More precisely, for $t>s$ the map $(t, s) \longmapsto U(t, s) \in \mathcal{L}(X)$ is continuous and continuously differentiable in $t, U(t, s)$ maps $X$ into $D(A(t))$ and it holds $\frac{\partial U(t, s)}{\partial t}=$ $A(t) U(t, s)$. Moreover, $U(t, s)$ and $(t-s) A(t) U(t, s)$ are exponentially bounded. We further have

$$
U(t, s) U(s, r)=U(t, r) \text { and } U(t, t)=I \text { for } t \geq s \geq r .
$$

Finally, for $s \in \mathbb{R}$ and $x \in \overline{D(A(s))}$, the function $t \longmapsto u(t)=U(t, s) x$ is continuous at $t=s$ and $u$ is the unique solution in $C([s, \infty), X) \cap C^{1}((s, \infty), X)$ of the Cauchy problem:

$$
u^{\prime}(t)=A(t) u(t), \quad t>s, \quad u(s)=x .
$$

For more details, we refer to $[1,2,23]$.

We introduce the interpolation and extrapolation spaces for the operators $A(t), t \in \mathbb{R}$. Let $A$ be a sectorial operator, i.e., $A$ satisfy (2.1) in place of $A(t)$ (it is well known that $A$ generates an analytic semigroup $\left(T_{A}(t)\right)_{t \geq 0}$ on $\left.X\right)$. For $\alpha \in(0,1)$, we use the real interpolation spaces:

$$
X_{\alpha}:=\overline{D(A)}{ }^{\|\cdot\|_{\alpha}}, \quad \text { where } \quad\|x\|_{\alpha}:=\sup _{\lambda>0}\left\|\lambda^{\alpha}(A-\omega) x\right\| \quad \text { for all } x \in D(A) .
$$

Then, $\left(X_{\alpha},\|\cdot\|_{\alpha}\right)$ are Banach spaces. Let $X_{0}:=X, X_{1}:=D(A)$ and $\|x\|_{0}=\|x\|,\|x\|_{1}=\|(A-\omega) x\|$ be the corresponding norms respectively. We also take the closed subspace $\hat{X}:=\overline{D(A)}$ of $X$. Furthermore, we define the extrapolation space denoted by $X_{-1}$ as the completion of $\hat{X}$ with respect to the norm $\|x\|_{-1}=$ $\|R(\omega, A) x\|$, for $x \in X$. This implies that $A$ has a unique continuous extension $A_{-1}: \hat{X} \longrightarrow X_{-1}$. Since for every $t \geq 0, T(t)$ commutes with the operator resolvent $R(\omega, A):=(A-\omega)^{-1}$, the extensions of $T_{A}(t)$ to $X_{-1}$ exist and define an analytic semigroup $\left(T_{A_{-1}}(t)\right)_{t \geq 0}$ generated by $A_{-1}$ with $D\left(A_{-1}\right)=\hat{X}$. We define the spaces:

$$
X_{\alpha-1}:=\left(X_{-1}\right)_{\alpha}=\bar{X}^{\|\cdot\|_{\alpha-1}} \quad \text { where } \quad\|x\|_{\alpha-1}:=\sup _{\lambda>0}\left\|\lambda^{\alpha} R\left(\lambda, A_{-1}-\omega\right) x\right\| .
$$


The restriction $A_{\alpha-1}: X_{\alpha} \longrightarrow X_{\alpha-1}$ of $A_{-1}$ generates the analytic semigroup $\left(T_{A_{\alpha-1}}(t)\right)_{t \geq 0}$ on $X_{\alpha-1}$ which is the extension of $T_{A}(t)$ on $X_{\alpha-1}$. Observe that $\omega-A_{\alpha-1}: X_{\alpha} \longrightarrow X_{\alpha-1}$ is an isometric isomorphism. We frequently use the continuous embeddings:

$$
\begin{array}{r}
D(A) \hookrightarrow X_{\beta} \hookrightarrow X_{\alpha} \hookrightarrow X \\
X \hookrightarrow X_{\beta-1} \hookrightarrow X_{\alpha-1} \hookrightarrow X_{-1}
\end{array}
$$

for all $0<\alpha<\beta<1$.

Let $A(t), t \in \mathbb{R}$ which satisfies (2.1), we set

$$
X_{\alpha}^{t}:=X_{\alpha}, \quad X_{\alpha-1}^{t}:=X_{\alpha-1}^{t} \quad \text { and } \quad \hat{X}^{t}:=\hat{X}
$$

where $A(t)$ is taken instead of $A$ in the definition of the spaces $X_{\delta}, 0<\delta<1, \hat{X}$ and the corresponding norms. Then, the embeddings in (2.3) hold and the norms of the embeddings are uniformly bounded in $t \in \mathbb{R}$.

Exponential dichotomy is another important tool in our study.

Definition 2.1 [3] An evolution family $(U(t, s))_{s \leq t}$ on a Banach space $X$ is called has an exponential dichotomy (or hyperbolic) in $\mathbb{R}$ if there exists a family of projections $P(t) \in \mathcal{L}(X), t \in \mathbb{R}$, being strongly continuous with respect to $t$, and constants $\delta, N>0$ such that

(i) $U(t, s) P(s)=P(t) U(t, s)$.

(ii) $U(t, s): Q(s) X \longrightarrow Q(t) X$ is invertible with the inverse $\tilde{U}(t, s)$.

(iii) $\|U(t, s) P(s)\| \leq N e^{-\delta(t-s)}$ and $\|\tilde{U}(s, t) Q(t)\| \leq N e^{-\delta(t-s)}$

for all $t, s \in \mathbb{R}$ with $s \leq t$, where, $Q(t):=I-P(t)$.

Definition 2.2 [3] Given a hyperbolic evolution family $(U(t, s))_{s \leq t}$, we define the Green function by:

$$
\Gamma(t, s)=\left\{\begin{aligned}
U(t, s) P(s), & t, s \in \mathbb{R}, s \leq t \\
-\tilde{U}(t, s) Q(s), & t, s \in \mathbb{R}, s>t .
\end{aligned}\right.
$$

Now, we give some dichotomy estimates of the extension evolution family $\left(U_{\alpha-1}(t, s)\right)_{s \leq t}$ of $(U(t, s))_{s \leq t}$ to the extrapolated spaces $X_{\alpha-1}^{t}, 0 \leq \alpha<1$.

Theorem 2.1 [5] Let $1-\mu<\alpha<1$. Assume that (2.1) and (2.2) hold. Then, the following holds for $s<t \leq s+t_{0}$ and $t_{0}>0$ :

The operators $U(t, s)$ have continuous extension $U_{\alpha-1}(t, s): X_{\alpha-1}^{s} \longrightarrow X$ satisfying

$$
\left\|U_{\alpha-1}(t, s) x\right\|_{\beta} \leq N\left(\alpha, t_{0}\right)(t-s)^{\alpha-\beta-1}\|x\|_{\alpha-1},
$$

and $U_{\alpha-1}(t, s) x=U_{\gamma-1}(t, s) x$ for $1-\mu<\gamma<\alpha<1,0 \geq \beta \geq 1$ and $x \in X_{\alpha-1}^{s}$.

Theorem 2.2 [5] Assume that (2.1) and (2.2) hold and that $U$ has an exponential dichotomy on $\mathbb{R}$. Let $1-\mu<\alpha \leq 1$. Then, the operators $P(t)$ and $Q(t)$ have continuous extensions $P_{\alpha-1}(t): X_{\alpha-1}^{t} \longrightarrow X_{\alpha-1}^{t}$ and $Q_{\alpha-1}(t): X_{\alpha-1}^{t} \longrightarrow X$ respectively, for $t \in \mathbb{R}$, which are uniformly bounded. Moreover, the following assertions hold for $t, s \in \mathbb{R}$ with $t \geq s$ :

(i) $Q_{\alpha-1}(t) X_{\alpha-1}^{t}=Q(t) X$.

(ii) $U_{\alpha-1}(t, s) P_{\alpha-1}(s)=P_{\alpha-1}(t) U_{\alpha-1}(t, s)$.

(iii) $U_{\alpha-1}(t, s): Q_{\alpha-1}(s) X_{\alpha-1}^{s} \longrightarrow Q_{\alpha-1}(t) X_{\alpha-1}^{t}$ is invertible with the inverse $\tilde{U}_{\alpha-1}(t, s)$.

(iv) Let $t, s \in \mathbb{R}, t \geq s$. Then,

$$
\begin{aligned}
\left\|U_{\alpha-1}(t, s) P_{\alpha-1}(s) x\right\| & \leq m(\alpha) \max \left\{(t-s)^{\alpha-1}, 1\right\} e^{-\delta(t-s)}\|x\|_{\alpha-1}, \quad x \in X_{\alpha-1}^{s} . \\
\left\|\tilde{U}_{\alpha-1}(t, s) Q_{\alpha-1}(t) x\right\| & \leq c(\alpha) e^{-\delta(t-s)}\|x\|_{\alpha-1}, \quad x \in X_{\alpha-1}^{t} .
\end{aligned}
$$


Theorem 2.3 Let $x \in X_{\alpha-1}, 0<\alpha \leq 1,0 \leq \beta \leq 1$ and $0 \leq \beta<\alpha$. Then, the following hold:

(i) There exists a constant $c(\alpha, \beta)$, such that

$$
\left\|\tilde{U}_{\alpha-1}(t, s) Q_{\alpha-1}(t) x\right\|_{\beta} \leq c(\alpha, \beta) e^{-\delta(t-s)}\|x\|_{\alpha-1} \quad \text { for } t \leq s .
$$

(ii) There exists a constant $m(\alpha, \beta)$, such that

$$
\left\|U_{\alpha-1}(t, s) P_{\alpha-1}(s) x\right\|_{\beta} \leq m(\alpha, \beta)(t-s)^{\alpha-\beta-1} e^{-\gamma(t-s)}\|x\|_{\alpha-1} \text { for } t \geq s .
$$

Proof. Let $x \in X_{\alpha-1}^{s}, 0<\alpha \leq 1,0 \leq \beta \leq 1$ and $0 \leq \beta<\alpha$.

(i) As $X_{\beta}$ is a space of class $J_{\beta}$, see [23, Definition 1.1.1], there exists a constant $n(\beta)$ such that

$$
\|x\|_{\beta} \leq n(\beta)\|x\|^{1-\beta}\|(\omega-A(t)) x\|^{\beta}, \quad x \in D(A(t)) .
$$

Hence, from (2.7), we obtain that

$$
\begin{aligned}
\left\|\tilde{U}_{\alpha-1}(t, s) Q_{\alpha-1}(t) x\right\|_{\beta} & \leq n(\beta)\left\|\tilde{U}_{\alpha-1}(t, s) Q_{\alpha-1}(t) x\right\|^{1-\beta}\left\|(A(t)-\omega) \tilde{U}_{\alpha-1}(t, s) Q_{\alpha-1}(t) x\right\|^{\beta} \\
& \leq n(\beta)\left\|\tilde{U}_{\alpha-1}(t, s) Q_{\alpha-1}(t) x\right\|^{1-\beta}\|(A(t)-\omega) Q(t)\|\left\|^{\beta}\right\| \tilde{U}_{\alpha-1}(t, s) Q_{\alpha-1}(t) x \|^{\beta} \\
& \leq n(\beta) c^{\beta}\left\|\tilde{U}_{\alpha-1}(t, s) Q_{\alpha-1}(t) x\right\| \\
& \leq c(\alpha, \beta) e^{-\delta(t-s)}\|x\|_{\alpha-1} \quad \text { for } t \leq s .
\end{aligned}
$$

(ii) Let $t-s>1$. Then, from the estimate (2.6), we obtain that

$$
\begin{aligned}
\left\|U_{\alpha-1}(t, s) P_{\alpha-1}(s) x\right\|_{\beta} & \leq\|U(t, t-1)\|_{\mathcal{L}\left(X, X_{\beta}^{t}\right)}\left\|U_{\alpha-1}(t-1, s) P_{\alpha-1}(s) x\right\| \\
& \leq m_{1}(\alpha, \beta)(t-s)^{\alpha-1} e^{-\delta(t-s)}\|x\|_{\alpha-1} \\
& \leq m_{1}(\alpha, \beta)(t-s)^{\beta} e^{-\frac{\delta}{2}(t-s)}(t-s)^{\alpha-\beta-1} e^{-\frac{\delta}{2}(t-s)}\|x\|_{\alpha-1} .
\end{aligned}
$$

Since $\tau^{\beta} e^{-\frac{\delta}{2} \tau} \rightarrow 0$ as $\tau \rightarrow \infty$. Then

$$
\left\|U_{\alpha-1}(t, s) P_{\alpha-1}(s) x\right\|_{\beta} \leq m_{1}(\alpha, \beta)(t-s)^{\alpha-\beta-1} e^{-\frac{\delta}{2}(t-s)}\|x\|_{\alpha-1} .
$$

Now, if $0 \leq t-s \leq 1$, it follows from (2.5) that

$$
\begin{aligned}
\left\|U_{\alpha-1}(t, s) P_{\alpha-1}(s) x\right\|_{\beta} & \leq N(\alpha, 1)(t-s)^{\alpha-\beta-1}\left\|P_{\alpha-1}(s) x\right\|_{\alpha-1} \\
& \leq c N(\alpha, 1) e^{\frac{\delta}{2}(t-s)}(t-s)^{\alpha-\beta-1}\|x\|_{\alpha-1} \\
& \leq m_{2}(\alpha, \beta)(t-s)^{\alpha-\beta-1} e^{-\frac{\delta}{2}(t-s)}\|x\|_{\alpha-1} \quad \text { for all } x \in X_{\alpha-1}^{s} .
\end{aligned}
$$

Therefore, there exist $m(\alpha, \beta)=\max \left\{m_{1}(\alpha, \beta), m_{2}(\alpha, \beta)\right\}>0$ and $\gamma:=\frac{\delta}{2}$ such that:

$$
\left\|U_{\alpha-1}(t, s) P_{\alpha-1}(s) x\right\|_{\beta} \leq m(\alpha, \beta)(t-s)^{\alpha-\beta-1} e^{-\frac{\delta}{2}(t-s)}\|x\|_{\alpha-1} \text { for } t \geq s .
$$

\subsection{Almost automorphic functions}

In this section, we recall some properties of almost automorphic functions in the classical sense and in Stepanov sense.

Definition 2.3 (H. Bohr) [9] A continuous function $f: \mathbb{R} \longrightarrow X$ is said to be almost periodic if for every $\varepsilon>0$, there exists $l_{\varepsilon}>0$, such that for every $a \in \mathbb{R}$, there exists $\tau \in\left[a, a+l_{\varepsilon}\right]$ satisfying:

$$
\|f(t+\tau)-f(t)\|<\varepsilon \quad \text { for all } t \in \mathbb{R} .
$$

The space of all such functions is denoted by $A P(\mathbb{R}, X)$. 
Definition 2.4 (S. Bochner) [8] A continuous function $f: \mathbb{R} \longrightarrow X$ is called almost automorphic if for every sequence $\left(\sigma_{n}\right)_{n \geq 0}$ of real numbers, there exist a subsequence $\left(s_{n}\right)_{n \geq 0} \subset\left(\sigma_{n}\right)_{n \geq 0}$ and a measurable function $g: \mathbb{R} \longrightarrow X$, such that

$$
g(t)=: \lim _{n} f\left(t+s_{n}\right) \quad \text { and } \quad f(t)=\lim _{n} g\left(t-s_{n}\right) \quad \text { for all } t \in \mathbb{R} .
$$

The space of all such functions is denoted by $A A(\mathbb{R}, X)$.

Then, we have the following inclusions:

$$
A P(\mathbb{R}, X) \subset A A(\mathbb{R}, X) \subset B C(\mathbb{R}, X) .
$$

Definition 2.5 A continuous function $F: \mathbb{R} \times \mathbb{R} \longrightarrow X$ is said to be bi-almost automorphic if for every sequence $\left(\sigma_{n}\right)_{n \geq 0}$ of real numbers, there exist a subsequence $\left(s_{n}\right)_{n \geq 0} \subset\left(\sigma_{n}\right)_{n \geq 0}$ and a measurable function $G: \mathbb{R} \times \mathbb{R} \longrightarrow X$, such that

$$
G(t, s)=: \lim _{n} F\left(t+s_{n}, s+s_{n}\right) \quad \text { and } \quad F(t, s)=\lim _{n} G\left(t-s_{n}, s-s_{n}\right) \quad \text { for all } t, s \in \mathbb{R} .
$$

The space of all such functions is denoted by $b A A(\mathbb{R}, X)$.

Proposition 2.1 [25] Let $f_{1}, f_{2}, f \in A A(\mathbb{R}, X)$ and $\lambda \in \mathbb{R}$. Then, the following are true:

(i) $\lambda f_{1}+f_{2} \in A A(\mathbb{R}, X)$.

(ii) The set $\{f(t): t \in \mathbb{R}\}$ is relatively compact in $X$.

(iii) The space $A A(\mathbb{R}, X)$ is translation invariant, i.e., for all $\tau \in \mathbb{R}, f \in A A(\mathbb{R}, X)$ implies $f(\cdot+\tau) \in$ $A A(\mathbb{R}, X)$.

(iv) The space $A A(\mathbb{R}, X)$ equipped with the supnorm is a Banach space.

(v) $f$ is bounded i.e., $\sup _{t \in \mathbb{R}}\|f(t)\|<\infty$.

\section{Remark 2.1}

(i) The function $g$ in Definition 2.4 is measurable not necessarily continuous. Using the characterization of $\mathrm{S}$. Bochner of almost periodicity one can claim easily that, if the convergence in Definition 2.4 is uniform in $t \in \mathbb{R}$, then necessarily $f$ belongs to $A P(\mathbb{R}, X)$.

(ii) An almost automorphic function may not be uniformly continuous. Indeed, the real function $f(t)=$ $\sin \left(\frac{1}{2+\cos (t)+\cos (\sqrt{2} t)}\right)$ for $t \in \mathbb{R}$, belong to $A A(\mathbb{R}, \mathbb{R})$, but is not uniformly continuous. Hence, $f$ does not belongs to $A P(\mathbb{R}, \mathbb{R})$.

Definition 2.6 [17] Let $1 \leq p<\infty$. A function $f \in L_{l o c}^{p}(\mathbb{R}, X)$ is said to be bounded in the sense of Stepanov if

$$
\sup _{t \in \mathbb{R}}\left(\int_{[t, t+1]}\|f(s)\|^{p} d s\right)^{\frac{1}{p}}=\sup _{t \in \mathbb{R}}\left(\int_{[0,1]}\|f(t+s)\|^{p} d s\right)^{\frac{1}{p}}<\infty .
$$

The space of all such functions is denoted by $B S^{p}(\mathbb{R}, X)$ and is provided with the following norm:

$$
\begin{aligned}
\|f\|_{B S^{p}} & :=\sup _{t \in \mathbb{R}}\left(\int_{[t, t+1]}\|f(s)\|^{p} d s\right)^{\frac{1}{p}} \\
& =\sup _{t \in \mathbb{R}}\|f(t+\cdot)\|_{L^{p}([0,1], X)} .
\end{aligned}
$$

Then, the following inclusions hold:

$$
B C(\mathbb{R}, X) \subset B S^{p}(\mathbb{R}, X) \subset L_{l o c}^{p}(\mathbb{R}, X) .
$$


Definition 2.7 (Bochner transform) [17] Let $f \in L_{l o c}^{p}(\mathbb{R}, X)$ for $1 \leq p<\infty$. The Bochner transform of $f$ is the function $f^{b}: \mathbb{R} \longrightarrow L^{p}([0,1], X)$ defined for all $t \in \mathbb{R}$ by

$$
\left(f^{b}(t)\right)(s)=f(t+s) \text { for } s \in[0,1] .
$$

Remark 2.2 [6] Let $f, g \in L_{l o c}^{p}(\mathbb{R}, X)$ for $1 \leq p<\infty$. Then, the following hold:

(i) $(f+g)^{b}=f^{b}+g^{b}$.

(ii) for all $\lambda \in \mathbb{R},(\lambda f)^{b}=\lambda f^{b}$.

(iii) for all $\tau \in \mathbb{R},\left(T_{\tau} f\right)^{b}=T_{\tau} f^{b}$, where $T_{\tau}$ is the translation map.

Now, we give the definition of almost automorphy in the sense of Stepanov.

Definition 2.8 [16] Let $1 \leq p<\infty$. A function $f \in L_{\text {loc }}^{p}(\mathbb{R}, X)$ is said to be almost automorphic in the sense of Stepanov (or $S^{p}$-almost automorphic), if for every sequence $\left(\sigma_{n}\right)_{n \geq 0}$ of real numbers, there exists a subsequence $\left(s_{n}\right)_{n \geq 0} \subset\left(\sigma_{n}\right)_{n \geq 0}$ and a measurable function $g \in L_{l o c}^{p}(\mathbb{R}, X)$, such that

$$
\lim _{n}\left(\int_{t}^{t+1}\left\|f\left(s+s_{n}\right)-g(s)\right\|^{p} d s\right)^{\frac{1}{p}}=0 \quad \text { and } \quad \lim _{n}\left(\int_{t}^{t+1}\left\|g\left(s-s_{n}\right)-f(s)\right\|^{p} d s\right)^{\frac{1}{p}} \quad \text { for all } t \in \mathbb{R} \text {. }
$$

The space of all such functions is denoted by $A A S^{p}(\mathbb{R}, X)$.

Theorem 2.4 [16] The following are equivalent:

(i) $f$ is $S^{p}$-almost automorphic in the sense of Definition 2.8 .

(ii) For every sequence $\left(\sigma_{n}\right)_{n \geq 0}$ of real numbers, there exists a subsequence $\left(s_{n}\right)_{n \geq 0} \subset\left(\sigma_{n}\right)_{n \geq 0}$

$$
\lim _{n, m}\left(\int_{t}^{t+1}\left\|f\left(\tau+s_{n}-s_{m}\right)-f(\tau)\right\|^{p} d \tau\right)^{\frac{1}{p}}=0
$$

for all $t \in \mathbb{R}$.

\section{Remark 2.3 [6]}

(i) Every almost automorphic function is $S^{p}$-almost automorphic for $1 \leq p<\infty$.

(ii) For all $1 \leq p_{1} \leq p_{2}<\infty$, if $f$ is $S^{p_{2}}$-almost automorphic, then $f$ is $S^{p_{1}}$-almost automorphic.

(iii) The Bochner transform of an $X$-valued function is a $L^{p}([0,1], X)$-valued function. Moreover, a function $f$ is $S^{p}$-almost automorphic if and only if $f^{b}$ is (Bochner) almost automorphic.

(iv) A function $\varphi(t, s)$ for $t \in \mathbb{R}, s \in[0,1]$ is the Bochner transform of a function $f$ (i.e., $\exists f: \mathbb{R} \longrightarrow X$ such that $\left.\left(f^{b}(t)\right)(s)=\varphi(t, s), t \in \mathbb{R}, s \in[0,1]\right)$ if and only if $\varphi(t+\tau, s-\tau)=\varphi(t, s)$ for all $t \in \mathbb{R}, s \in[0,1]$ and $\tau \in[s-1, s]$.

Proposition $2.2[16]$ For all $1 \leq p<\infty,\left(A A S^{p}(\mathbb{R}, X),\|\cdot\|_{B S^{p}}\right)$ is a Banach space.

\section{$2.3 \mu$-pseudo almost automorphic functions}

This section is provided to properties of $\mu$-ergodic and $\mu$-pseudo almost automorphic functions. In the sequel, we denote by $\mathcal{B}(\mathbb{R})$ the Lebesgue $\sigma$-field of $\mathbb{R}$ and by $\mathcal{M}$ the set of all positive measures $\mu$ on $\mathcal{B}(\mathbb{R})$ satisfying $\mu(\mathbb{R})=+\infty$ and $\mu([a, b])<+\infty$ for all $a, b \in \mathbb{R}$ with $(a \leq b)$, we denote also by $Y$ any other Banach space. We assume the following hypothesis.

(H2) For all $\tau \in \mathbb{R}$, there exist $\beta>0$ and a bounded interval $I$ such that

$$
\mu(\{a+\tau: a \in A\}) \leq \beta \mu(A) \quad \text { where } A \in \mathcal{B}(\mathbb{R}) \text { and } A \cap I=\varnothing .
$$


Definition 2.9 [12] Let $\mu \in \mathcal{M}$. A continuous bounded function $f: \mathbb{R} \longrightarrow X$ is called $\mu$-ergodic, if

$$
\lim _{r \rightarrow+\infty} \frac{1}{\mu([-r, r])} \int_{[-r, r]}\|f(t)\| d \mu(t)=0 .
$$

The space of all such functions is denoted by $\mathcal{E}(\mathbb{R}, X, \mu)$.

Proposition 2.3 [12] Let $\mu \in \mathcal{M}$. Then,

(i) $\left.\mathcal{E}(\mathbb{R}, X, \mu),\|\cdot\|_{\infty}\right)$ is a Banach space.

(ii) If $\mu$ satisfies (H2), then $\mathcal{E}(\mathbb{R}, X, \mu)$ is translation invariant.

\section{Examples 2.1}

(1) An ergodic function in the sense of Zhang [32] is a $\mu$-ergodic function in the particular case where the measure $\mu$ is the Lebesgue measure.

(2) Let $\rho: \mathbb{R} \longrightarrow[0,+\infty)$ be a $\mathcal{B}(\mathbb{R})$-measurable function. We define the positive measure $\mu$ on $\mathcal{B}(\mathbb{R})$ by

$$
\mu(A)=\int_{A} \rho(t) d t \quad \text { for } A \in \mathcal{B}(\mathbb{R}),
$$

where $d t$ denotes the Lebesgue measure on $\mathcal{B}(\mathbb{R})$. The measure $\mu$ is absolutely continuous with respect to $d t$ and the function $\rho$ is called the Radon-Nikodym derivative of $\mu$ with respect to $d t$. In this case $\mu \in \mathcal{M}$ if and only if the function $\rho$ is locally Lebesgue-integrable on $\mathbb{R}$ and it satisfies

$$
\int_{\mathbb{R}} \rho(t) d t=+\infty
$$

(3) In [21], the authors considered the space of bounded continuous functions $f: \mathbb{R} \longrightarrow X$ satisfying

$$
\lim _{r \rightarrow+\infty} \frac{1}{2 r} \int_{[-r, r]}\|f(t)\| d t=0 \text { and } \lim _{N \rightarrow+\infty} \frac{1}{2 N+1} \sum_{n=-N}^{N}\|f(n)\|=0 .
$$

This space coincides with the space of $\mu$-ergodic functions where $\mu$ is defined in $\mathcal{B}(\mathbb{R})$ by the sum $\mu(A)=$ $\mu_{1}(A)+\mu_{2}(A)$ with $\mu_{1}$ is the Lebesgue measure on $(\mathbb{R}, \mathcal{B}(\mathbb{R}))$ and

$$
\mu_{2}(A)=\left\{\begin{aligned}
\operatorname{card}(A \cap \mathbb{Z}) & \text { if } A \cap \mathbb{Z} \text { is finite } \\
\infty & \text { if } A \cap \mathbb{Z} \text { is infinite. }
\end{aligned}\right.
$$

Definition 2.10 [11] Let $\mu \in \mathcal{M}$. A continuous function $f: \mathbb{R} \longrightarrow X$ is said to be $\mu$-pseudo almost automorphic if $f$ is written in the form:

$$
f=g+\varphi,
$$

where $g \in A A(\mathbb{R}, X)$ and $\varphi \in \mathcal{E}(\mathbb{R}, X, \mu)$.

The space of all such functions is denoted by $P A A(\mathbb{R}, X, \mu)$.

Proposition 2.4 [11] Let $\mu \in \mathcal{M}$ satisfy (H2). Then the following are true:

(i) The decomposition of a $\mu$-pseudo almost automorphic in the form $f=g+\varphi$ where $g \in A A(\mathbb{R}, X)$ and $\varphi \in \mathcal{E}(\mathbb{R}, X, \mu)$, is unique.

(ii) $P A A(\mathbb{R}, X, \mu)$ equipped with the supnorm is a Banach space.

(iii) $P A A(\mathbb{R}, X, \mu)$ is invariant by translation, that is:

$$
f \in P A A(\mathbb{R}, X, \mu) \quad \text { implies } \quad f_{\tau} \in P A A(\mathbb{R}, X, \mu) \quad \text { for all } \tau \in \mathbb{R} .
$$

Now, we give the definition and the important properties of $\mu$-S ${ }^{p}$-pseudo almost automorphic functions.

Definition 2.11 [17] Let $\mu \in \mathcal{M}$. A function $f \in B S^{p}(\mathbb{R}, X)$ is said to be $\mu$-ergodic in the sense of Stepanov (or $\mu$-S ${ }^{p}$-ergodic) if

$$
\lim _{r \rightarrow+\infty} \frac{1}{\mu([-r, r])} \int_{[-r, r]}\left(\int_{[t, t+1]}\|f(s)\|^{p} d s\right)^{\frac{1}{p}} d \mu(t)=\lim _{r \rightarrow+\infty} \frac{1}{\mu([-r, r])} \int_{[-r, r]}\left\|f^{b}(t)\right\|_{p, X} d \mu(t)=0 .
$$

The space of all such functions is denoted by $\mathcal{E}^{p}(\mathbb{R}, X, \mu)$. 
Remark 2.4 Using (2.13) we obtain that, $f \in \mathcal{E}^{p}(\mathbb{R}, X, \mu)$ if and only if $f^{b} \in \mathcal{E}\left(\mathbb{R}, L^{p}([0,1], X), \mu\right)$.

Proposition 2.5 [6] Let $\mu \in \mathcal{M}$. Then, for all $1 \leq p<\infty,\left(\mathcal{E}^{p}(\mathbb{R}, X, \mu),\|\cdot\|_{B S^{p}}\right)$ is a Banach space.

Proposition 2.6 [17] Let $\mu \in \mathcal{M}$ satisfy (H2). Then, the following hold:

(i) $\mathcal{E}^{p}(\mathbb{R}, X, \mu)$ is translation invariant.

(ii) $\mathcal{E}(\mathbb{R}, X, \mu) \subset \mathcal{E}^{p}(\mathbb{R}, X, \mu)$.

Example 2.1 Let $f: \mathbb{R} \longrightarrow \mathbb{R}$ defined by:

$$
f(t)= \begin{cases}\frac{1}{k} & k \leq t \leq k+\frac{1}{2 k} \text { with } k \in \mathbb{N}^{*}, \\ 0 & \text { otherwise. }\end{cases}
$$

The function $f$ is not continuous in $\mathbb{R}$, which implies that $f \notin \mathcal{E}(\mathbb{R}, \mathbb{R}, \mu)$. But, $f \in \mathcal{E}^{1}(\mathbb{R}, \mathbb{R}, \mu)$. In fact, let $r>1$. Then, we have

$$
\begin{aligned}
\frac{1}{2 r} \int_{-r}^{r} \int_{t}^{t+1} f(s) d s d t & \leq \frac{1}{2 r} \int_{1}^{+\infty} \int_{t}^{t+1} f(s) d s d t \\
& \leq \frac{1}{2 r} \int_{1}^{+\infty} \int_{[t]}^{[t]+2} f(s) d s d t \\
& \leq \frac{1}{2 r} \sum_{k \geq 1} \frac{1}{k^{2}}=\frac{\pi^{2}}{12 r} \rightarrow 0 \text { as } r \rightarrow+\infty
\end{aligned}
$$

In the next, we give a sufficient condition for a $\mu$-S $S^{p}$-ergodic function to be $\mu$-ergodic.

Proposition 2.7 [17] Let $\mu \in \mathcal{M}$ satisfy (H2) and $f: \mathbb{R} \longrightarrow X$ be bounded, uniformly continuous and $\mu$-S $S^{p}$-ergodic. Then, $f$ is $\mu$-ergodic.

Definition 2.12 [17] Let $\mu \in \mathcal{M}$. A function $f \in B S^{p}(\mathbb{R}, X)$ is said to be $\mu$-pseudo almost automorphic in the sense of Stepanov (or $\mu$-S $S^{p}$-pseudo almost automorphic) if:

$$
f=\tilde{f}+\varphi,
$$

where $\tilde{f} \in A A S^{p}(\mathbb{R}, X)$ and $\varphi \in \mathcal{E}^{p}(\mathbb{R}, X, \mu)$.

The space of all such functions will be denoted $\operatorname{PAAS}^{p}(\mathbb{R}, X, \mu)$.

Definition 2.13 [12] Let $\mu \in \mathcal{M}$. A continuous function $f: \mathbb{R} \times X \longrightarrow Y$ is said to be $\mu$-pseudo almost automorphic if $f$ is written in the form:

$$
f=g+\varphi,
$$

where $g \in A A U(\mathbb{R} \times X, Y)$, and $\varphi \in \mathcal{E} U(\mathbb{R} \times X, Y, \mu)$.

The space of all such functions is denoted by $P A A U(\mathbb{R} \times X, Y, \mu)$.

Definition 2.14 Let $\mu \in \mathcal{M}$ and $f: \mathbb{R} \times X \longrightarrow Y$ be such that $f(\cdot, x) \in B S^{p}(\mathbb{R}, Y)$ for each $x \in X$. The function $f$ is $\mu$-S $S^{p}$-almost automorphic if $f$ is written as:

$$
f=g+\varphi,
$$

where $g \in A A S^{p} U(\mathbb{R} \times X, Y)$, and $\varphi \in \mathcal{E}^{p} U(\mathbb{R} \times X, Y, \mu)$.

The space of all such functions will be denoted $P A A S^{p} U(\mathbb{R}, X, \mu)$.

\section{New composition results of $\mu-S^{p}$-pseudo almost automorphic func- tions}

In this section, we prove a new composition result of $\mu$-S ${ }^{p}$-pseudo almost automorphic functions using the uniform continuity property. 
Lemma 3.1 Let $1 \leq p<+\infty$ and $f: \mathbb{R} \times X \longrightarrow Y$ be a function such that $f(\cdot, x) \in L_{\text {loc }}^{p}(\mathbb{R}, Y)$ for each $x \in X$. Then, $f \in A A S^{p} U(\mathbb{R} \times X, Y)$ if and only if the following hold:

(i) For each $x \in X, f(\cdot, x) \in A A S^{p}(\mathbb{R}, Y)$.

(ii) $f$ is $S^{p}$-uniformly continuous with respect to the second argument on each compact subset $K$ in $X$, namely: for all $\varepsilon>0$ there exists $\delta_{K, \varepsilon}$ such that for all $x_{1}, x_{2} \in K$, we have

$$
\left\|x_{1}-x_{2}\right\| \leq \delta_{K, \varepsilon} \Longrightarrow\left(\int_{t}^{t+1}\left\|f\left(s, x_{1}\right)-f\left(s, x_{2}\right)\right\|_{Y}^{p} d s\right)^{\frac{1}{p}} \leq \varepsilon \quad \text { for all } t \in \mathbb{R} .
$$

Proof. Let $f \in A A S^{p} U(\mathbb{R} \times X, Y)$ and $f^{b}: \mathbb{R} \times X \longrightarrow L^{p}([0,1], Y)$ be the Bochner transform associated to $f$. It follows in view of [11, Proposition 5.5], that (i) is clearly satisfied and that: for each compact subset $K$ in $X$, for all $\varepsilon>0$ there exists $\delta_{K, \varepsilon}$ such that for all $x_{1}, x_{2} \in K$, we have

$$
\left\|x_{1}-x_{2}\right\| \leq \delta_{K, \varepsilon} \Longrightarrow\left\|f^{b}\left(t, x_{1}\right)-f^{b}\left(t, x_{2}\right)\right\|_{Y, p} \leq \varepsilon \quad \text { for all } t \in \mathbb{R} .
$$

Since

$$
\begin{aligned}
\left\|f^{b}\left(t, x_{1}\right)-f^{b}\left(t, x_{2}\right)\right\|_{Y, p} & =\left(\int_{[0,1]}\left\|\left(f^{b}\left(t, x_{1}\right)\right)(s)-\left(f^{b}\left(t, x_{2}\right)\right)(s)\right\|_{Y}^{p} d s\right)^{\frac{1}{p}} \\
& =\left(\int_{t}^{t+1}\left\|f\left(s, x_{1}\right)-f\left(s, x_{2}\right)\right\|_{Y}^{p} d s\right)^{\frac{1}{p}} \quad \text { for all } t \in \mathbb{R} .
\end{aligned}
$$

It follows that (3.1) holds and then (ii) is achieved.

Conversely, let $f: \mathbb{R} \times X \longrightarrow Y$ be a function such that $f(\cdot, x) \in L_{\text {loc }}^{p}(\mathbb{R}, Y)$ for each $x \in X$. Assume that $f$ satisfies (i)-(ii). Let us fix a compact subset $K$ in $X$ and $\varepsilon>0$. Since $K$ is compact, it follows that there exists a finite subset $\left\{x_{1}, \ldots, x_{n}\right\} \subset K\left(n \in \mathbb{N}^{*}\right)$ such that $K \subseteq \bigcup_{i=1}^{n} B\left(x_{i}, \delta_{K, \varepsilon}\right)$. Therefore, for $x \in K$, there exist $i=1, \ldots, n$ satisfying $\left\|x-x_{i}\right\| \leq \delta_{K, \varepsilon}$. Let $\left(\sigma_{n}\right)_{n \geq 0}$ be a sequence of real numbers and let $\left(s_{n}\right)_{n \geq 0} \subset\left(\sigma_{n}\right)_{n \geq 0}$ be a subsequence such that:

$$
\begin{aligned}
& \left(\int_{t}^{t+1}\left\|f\left(s+s_{l}-s_{k}, x\right)-f(s, x)\right\|_{Y}^{p} d s\right)^{\frac{1}{p}} \leq\left(\int_{t}^{t+1}\left\|f\left(s+s_{l}-s_{k}, x\right)-f\left(s+s_{l}-s_{k}, x_{i}\right)\right\|_{Y}^{p} d s\right)^{\frac{1}{p}} \\
& +\left(\int_{t}^{t+1}\left\|f\left(s+s_{l}-s_{k}, x_{i}\right)-f\left(s, x_{i}\right)\right\|_{Y}^{p} d s\right)^{\frac{1}{p}}+\left(\int_{t}^{t+1}\left\|f\left(s, x_{i}\right)-f(s, x)\right\|_{Y}^{p} d s\right)^{\frac{1}{p}} \text { for all } t \in \mathbb{R} .(3.2)
\end{aligned}
$$

From (i), $f\left(\cdot, x_{i}\right) \in A A S^{p}(\mathbb{R}, Y)$. Hence, for $k, l$ large enough

$$
\left(\int_{t}^{t+1}\left\|f\left(s+s_{l}-s_{k}, x_{i}\right)-f\left(s, x_{i}\right)\right\|_{Y}^{p} d s\right)^{\frac{1}{p}} \leq \frac{\varepsilon}{3} \quad \text { for all } t \in \mathbb{R} .
$$

Otherwise, since $\left\|x-x_{i}\right\| \leq \delta_{K, \varepsilon}$ and by using (ii) we claim that

$$
\left(\int_{t}^{t+1}\left\|f\left(s+s_{l}-s_{k}, x\right)-f\left(s+s_{l}-s_{k}, x_{i}\right)\right\|_{Y}^{p} d s\right)^{\frac{1}{p}} \leq \frac{\varepsilon}{3} \quad \text { for all } t \in \mathbb{R} \text { and } k, l \in \mathbb{N}
$$

and

$$
\left(\int_{t}^{t+1}\left\|f(s, x)-f\left(s, x_{i}\right)\right\|_{Y}^{p} d s\right)^{\frac{1}{p}} \leq \frac{\varepsilon}{3} \quad \text { for all } t \in \mathbb{R} .
$$

Consequently, we replace (3.3), (3.4) and (3.5) in (3.2), we obtain, for $k, l$ large enough, that

$$
\sup _{x \in K}\left(\int_{t}^{t+1}\left\|f\left(s+s_{l}-s_{k}, x\right)-f(s, x)\right\|_{Y}^{p} d s\right)^{\frac{1}{p}} \leq \varepsilon \text { for all } t \in \mathbb{R} .
$$


By Lemma 3.1, we deduce the following result.

Proposition 3.1 Let $\mu \in \mathcal{M}$ and $f \in P A A S^{p} U(\mathbb{R} \times X, Y, \mu)$, for $1 \leq p<+\infty$. Then, the following hold:

(i) For each $x \in X, f(\cdot, x) \in A A S^{p}(\mathbb{R}, Y, \mu)$.

(ii) $f$ is $S^{p}$-uniformly continuous with respect to the second argument on each compact subset $K$ in $X$ in the following sense: for all $\varepsilon>0$ there exists $\delta_{K, \varepsilon}$ such that for all $x_{1}, x_{2} \in K$, one has:

$$
\left\|x_{1}-x_{2}\right\| \leq \delta_{K, \varepsilon} \Longrightarrow\left(\int_{t}^{t+1}\left\|f\left(s, x_{1}\right)-f\left(s, x_{2}\right)\right\|_{Y}^{p} d s\right)^{\frac{1}{p}} \leq \varepsilon \quad \text { for all } t \in \mathbb{R} .
$$

Theorem 3.1 Let $1 \leq p<+\infty$ and $f \in A A S^{p} U(\mathbb{R} \times X, Y)$. Assume that $u \in A A(\mathbb{R}, X)$.

Then, $f(\cdot, u(\cdot)) \in A A S^{p}(\mathbb{R}, Y)$.

Proof. Let $\left(\sigma_{n}\right)_{n \geq 0}$ be a sequence of real numbers, since $f \in A A S^{p} U(\mathbb{R} \times X, Y)$, then for each $x \in X$ there exists a subsequence $\left(s_{n}\right)_{n \geq 0}$ such that

$$
\left(\int_{t}^{t+1}\left\|f\left(s+s_{l}-s_{k}, x\right)-f(s, x)\right\|_{Y}^{p} d s\right)^{\frac{1}{p}} \rightarrow 0 \text { as } k, l \rightarrow \infty
$$

for all $t \in \mathbb{R}$. On the other hand, we have

$$
\begin{aligned}
& \left(\int_{t}^{t+1}\left\|f\left(s+s_{l}-s_{k}, u\left(s+s_{l}-s_{k}\right)\right)-f(s, u(s))\right\|_{Y}^{p} d s\right)^{\frac{1}{p}} \leq \\
& \left(\int_{t}^{t+1}\left\|f\left(s+s_{l}-s_{k}, u\left(s+s_{l}-s_{k}\right)\right)-f\left(s+s_{l}-s_{k}, u(s)\right)\right\|_{Y}^{p} d s\right)^{\frac{1}{p}} \\
& +\left(\int_{t}^{t+1}\left\|f\left(s+s_{l}-s_{k}, u(s)\right)-f(s, u(s))\right\|_{Y}^{p} d s\right)^{\frac{1}{p}} \quad \text { for all } t \in \mathbb{R} .
\end{aligned}
$$

Moreover, given $K:=\overline{\{u(t): t \in \mathbb{R}\}}$ a compact subset of $X$ and $\varepsilon>0$. Using Lemma 3.1-(ii) it follows that there exists $\delta_{\varepsilon, K}>0$ such that (3.1) holds. Since $u \in A A(\mathbb{R}, X)$. Then, $u(s) \in K$ for all $s \in \mathbb{R}$ and for $k, l$ large enough, $\left\|u\left(s+s_{l}-s_{k}\right)-u(s)\right\| \leq \delta$ for each $s \in \mathbb{R}$ which implies that

$$
\left(\int_{t}^{t+1}\left\|f\left(s+s_{l}-s_{k}, u\left(s+s_{l}-s_{k}\right)\right)-f\left(s+s_{l}-s_{k}, u(s)\right)\right\|_{Y}^{p} d s\right)^{\frac{1}{p}} \leq \frac{\varepsilon}{4} \quad \text { for all } t \in \mathbb{R} .
$$

Since $K$ is compact, there exist a finite subset $\left\{u_{1}, \ldots, u_{n}\right\} \subset K\left(n \in \mathbb{N}^{*}\right)$ such that $K \subseteq \bigcup_{i=1}^{n} B\left(u_{i}, \delta_{K, \varepsilon}\right)$. Then, for all $t \in \mathbb{R}$, there exists $i(t)=1, \ldots, n$ such that $\left\|u(t)-u_{i(t)}\right\| \leq \delta_{K, \varepsilon}$. Thus

$$
\left(\int_{t}^{t+1}\left\|f\left(s+s_{l}-s_{k}, u(s)\right)-f\left(s+s_{l}-s_{k}, u_{i(t)}\right)\right\|_{Y}^{p} d s\right)^{\frac{1}{p}} \leq \frac{\varepsilon}{4}
$$

and

$$
\left(\int_{t}^{t+1}\left\|f(s, u(s))-f\left(s, u_{i(t)}\right)\right\|_{Y}^{p} d s\right)^{\frac{1}{p}} \leq \frac{\varepsilon}{4}
$$

Using 3.1-(i), we get that

$$
\left(\int_{t}^{t+1}\left\|f\left(s+s_{l}-s_{k}, u_{i(t)}\right)-f\left(s, u_{i(t)}\right)\right\|_{Y}^{p} d s\right)^{\frac{1}{p}} \leq \frac{\varepsilon}{4}
$$

Consequently, by (3.8), (3.9), (3.10) and (3.11), we obtain for $l, k$ large enough, that

$$
\left(\int_{t}^{t+1}\left\|f\left(s+s_{l}-s_{k}, u\left(s+s_{l}-s_{k}\right)\right)-f(s, u(s))\right\|_{Y}^{p} d s\right)^{\frac{1}{p}} \leq \frac{\varepsilon}{4}+\frac{\varepsilon}{4}+\frac{\varepsilon}{4}+\frac{\varepsilon}{4}=\varepsilon \quad \text { for all } t \in \mathbb{R} .
$$


Theorem 3.2 Let $\mu \in \mathcal{M}$. If $u \in B S^{p}(\mathbb{R}, X)$ with $\left.K=\overline{\{u(t): t \in \mathbb{R}}\right\}$ is compact in $X$ and $f \in \mathcal{E}^{p} U(\mathbb{R} \times$ $X, Y, \mu)$. Then, $f(\cdot, u(\cdot)) \in \mathcal{E}^{p}(\mathbb{R}, Y, \mu)$.

Proof. Let $f \in \mathcal{E}^{p} U(\mathbb{R} \times X, Y, \mu)$ and $\left.K=\overline{\{u(t): t \in \mathbb{R}}\right\}$ be fixed. Then for all $\varepsilon>0$ there exists $\delta_{\varepsilon, K}>0$ such that (3.1) holds. Since $K$ is compact, then there exists a finite subset $\left\{u_{1}, \ldots, u_{n}\right\} \subset K\left(n \in \mathbb{N}^{*}\right)$ such that $K \subseteq \bigcup_{i=1}^{n} B\left(u_{i}, \delta_{K, \varepsilon}\right)$. Therefore, for all $t \in \mathbb{R}$, there exists $i(t)=1, \ldots, n$ such that $\left\|u(t)-u_{i(t)}\right\| \leq \delta_{K, \varepsilon}$.

$$
\begin{aligned}
\left(\int_{t}^{t+1}\|f(s, u(s))\|_{Y}^{p} d s\right)^{\frac{1}{p}} & \leq\left(\int_{t}^{t+1}\left\|f(s, u(s))-f\left(s, u_{i(t)}\right)\right\|_{Y}^{p} d s\right)^{\frac{1}{p}}+\left(\int_{t}^{t+1}\left\|f\left(s, u_{i(t)}\right)\right\|_{Y}^{p} d s\right)^{\frac{1}{p}} \\
& \leq \varepsilon+\sum_{i=1}^{n}\left(\int_{t}^{t+1}\left\|f\left(s, u_{i}\right)\right\|_{Y}^{p} d s\right)^{\frac{1}{p}} .
\end{aligned}
$$

Since for $i=1, \ldots, n, f\left(\cdot, x_{i}\right) \in \mathcal{E}^{p}(\mathbb{R}, Y, \mu)$. Then, for $r>0$, we have

$$
\frac{1}{\mu([-r, r])} \int_{-r}^{r}\left(\int_{t}^{t+1}\|f(s, u(s))\|_{Y}^{p} d s\right)^{\frac{1}{p}} d \mu(t) \leq \varepsilon+\frac{1}{\mu([-r, r])} \sum_{i=1}^{n} \int_{-r}^{r}\left(\int_{t}^{t+1}\left\|f\left(s, u_{i}\right)\right\|_{Y}^{p} d s\right)^{\frac{1}{p}} d \mu(t) .
$$

Consequently,

$$
\limsup _{r \rightarrow+\infty} \frac{1}{\mu([-r, r])} \int_{-r}^{r}\left(\int_{t}^{t+1}\|f(s, u(s))\|_{Y}^{p} d s\right)^{\frac{1}{p}} d \mu(t) \leq \varepsilon .
$$

Since formula (3.13) holds for all $\varepsilon>0$, we obtain that

$$
\lim _{r \rightarrow+\infty} \frac{1}{\mu([-r, r])} \int_{-r}^{r}\left(\int_{t}^{t+1}\|f(s, u(s))\|_{Y}^{p} d s\right)^{\frac{1}{p}} d \mu(t)=0 .
$$

Remark 3.1 From (3.15), we can deduce that for all $1 \leq p<\infty, f(\cdot, u(\cdot)) \in B S^{p}(\mathbb{R}, Y)$.

Corollary 3.1 Let $\mu \in \mathcal{M}$. Assume that $u \in A A(\mathbb{R}, X)$ and $f \in \mathcal{E}^{p} U(\mathbb{R} \times X, Y, \mu)$. Then, $f(\cdot, u(\cdot)) \in$ $\mathcal{E}^{p}(\mathbb{R}, Y, \mu)$.

Proof. From $u \in A A(\mathbb{R}, X)$, we deduce that $u \in A P S^{p}(\mathbb{R}, X)$ and that $\left.K=\overline{\{u(t): t \in \mathbb{R}}\right\}$ is compact in $X$. Hence, conditions and hypotheses of Theorem 3.2 are satisfied.

Lemma 3.2 [12] Let $\mu \in \mathcal{M}$ and $f \in B C(\mathbb{R}, X)$. Then, $f \in \mathcal{E}(\mathbb{R}, X, \mu)$ if and only if for all $\varepsilon>0$

$$
\lim _{r \rightarrow+\infty} \frac{\mu\left(M_{\varepsilon, r}(f)\right)}{\mu([-r, r])}=0,
$$

where $M_{\varepsilon, r}(f):=\{t \in[-r, r]:\|f(t)\| \geq \varepsilon\}$.

The proof of our result of composition of $\mu$-S $S^{p}$-pseudo almost automorphic functions is based on the following Lemma due to Schwartz [28, p. 109].

Lemma 3.3 Let $\Phi \in C(X, Y)$. Then, for each compact $K \subset X$ and for all $\varepsilon>0$, there exists $\delta_{K, \varepsilon}>0$, such that for any $x_{1}, x_{2} \in X$, one has

$$
x_{1} \in K \quad \text { and } \quad\left\|x_{1}-x_{2}\right\| \leq \delta \Rightarrow\left\|\Phi\left(x_{1}\right)-\Phi\left(x_{2}\right)\right\|_{Y} \leq \varepsilon .
$$

Theorem 3.3 Let $\mu \in \mathcal{M}$ and $f: \mathbb{R} \times X \longrightarrow Y$. Assume that:

(i) $f=\tilde{f}+\varphi \in P A A S^{p} U(\mathbb{R} \times X, Y, \mu)$ with $\tilde{f} \in A A S^{p} U(\mathbb{R} \times X, Y)$ and $\varphi \in \mathcal{E}^{p} U(\mathbb{R} \times X, Y, \mu)$.

(ii) $u=u_{1}+u_{2} \in P A A(\mathbb{R}, X, \mu)$, where $u_{1} \in A A(\mathbb{R}, X)$ and $u_{2} \in \mathcal{E}(\mathbb{R}, X, \mu)$.

(iii) For every bounded subset $B \subset X$ the set $\Lambda:=\{f(\cdot, x): x \in B\}$ is bounded in $B S^{p}(\mathbb{R}, X)$.

Then, $f(\cdot, u(\cdot)) \in P A A S^{p}(\mathbb{R}, Y, \mu)$. 


\section{Remark 3.2}

The condition (iii) is needed only to prove that $f(\cdot, u(\cdot)) \in B S^{p}(\mathbb{R}, Y)$.

Proof of Theorem 3.3. By definition of $f$ and $u$, we obtain the following decomposition:

$$
f(t, u(t))=\underbrace{\tilde{f}\left(t, u_{1}(t)\right)}_{\tilde{F}(t)}+\underbrace{\left[f(t, u(t))-f\left(t, u_{1}(t)\right)\right]}_{F(t)}+\underbrace{\varphi\left(t, u_{1}(t)\right)}_{\Psi(t)} .
$$

Using Theorem 3.1, it follows that $\tilde{F} \in A A S^{p}(\mathbb{R}, Y)$ and from Corollary 3.1, we deduce that $\Psi \in \mathcal{E}^{p}(\mathbb{R}, Y, \mu)$. Now, it suffices to prove that $F \in \mathcal{E}^{p}(\mathbb{R}, Y, \mu)$. In view of Lemma 3.2, we have for all $\varepsilon>0$,

$$
\lim _{r \rightarrow+\infty} \frac{\mu\left(M_{\varepsilon, r}\left(u_{2}\right)\right)}{\mu([-r, r])}=0
$$

Let $\varepsilon>0$. Then, for $r>0$, we have

$$
\begin{aligned}
& \frac{1}{\mu([-r, r])} \int_{-r}^{r}\left(\int_{t}^{t+1}\|F(s)\|_{Y}^{p} d s\right)^{\frac{1}{p}} d \mu(t) \\
\leq & \frac{1}{\mu([-r, r])} \int_{M_{\varepsilon, r}\left(u_{2}\right)}\left(\int_{t}^{t+1}\|F(s)\|_{Y}^{p} d s\right)^{\frac{1}{p}} d \mu(t)+\frac{1}{\mu([-r, r])} \int_{[-r, r] \backslash M_{\varepsilon, r}\left(u_{2}\right)}\left(\int_{t}^{t+1}\|F(s)\|_{Y}^{p} d s\right)^{\frac{1}{p}} d \mu(t) \\
\leq & \|F\|_{B S^{p}} \frac{\mu\left(M_{\varepsilon, r}\left(u_{2}\right)\right)}{\mu([-r, r])}+\frac{1}{\mu([-r, r])} \int_{[-r, r] \backslash M_{\varepsilon, r}\left(u_{2}\right)}\left(\int_{t}^{t+1}\left\|f(s, u(s))-f\left(s, u_{1}(s)\right)\right\|^{p} d s\right)^{\frac{1}{p}} d \mu(t) . \quad(3.17)
\end{aligned}
$$

Let $K=\overline{\left\{u_{1}(t): t \in \mathbb{R}\right\}}$. Since $u_{1} \in A A(\mathbb{R}, X)$, then $K$ is a compact subset of $X$. Hence, we define the function:

$$
\Phi: X \longrightarrow P A A S^{p}(\mathbb{R}, Y) ; x \mapsto f(\cdot, x) .
$$

Since $f \in P A A S^{p} U(\mathbb{R} \times X, Y, \mu)$, then, from Proposition 3.1, we deduce that the restriction of $\Phi$ on all compact $K$ of $X$ is uniformly continuous, which is equivalent to say that the function $\Phi$ is continuous on $X$. From Lemma 3.3 applied to $\Phi$, we deduce that for $\varepsilon>0$, there exists $\delta>0$ such that, for all $\xi_{1}, \xi_{2} \in X$, we have

$$
\xi_{1} \in K \quad \text { and } \quad\left\|\xi_{1}-\xi_{2}\right\| \leq \delta \Rightarrow\left(\int_{t}^{t+1}\left\|f\left(s, \xi_{1}\right)-f\left(s, \xi_{2}\right)\right\|_{Y}^{p} d s\right)^{\frac{1}{p}} \leq \varepsilon \quad \text { for all } t \in \mathbb{R} .
$$

Then, from $u(t)=u_{1}(t)+u_{2}(t)$ and $u_{1}(t) \in K$ for $t \in \mathbb{R}$, we have

$$
t \in \mathbb{R} \quad \text { and } \quad\left\|u_{2}(s)\right\| \leq \delta \quad \text { for } s \in[t, t+1] \Rightarrow\left(\int_{t}^{t+1}\left\|f(s, u(s))-f\left(s, u_{1}(s)\right)\right\|_{Y}^{p} d s\right)^{\frac{1}{p}} \leq \varepsilon
$$

Therefore, by the fact that $u_{2} \in \mathcal{E}(\mathbb{R}, X, \mu)$, we have

$$
\limsup _{r \rightarrow+\infty} \frac{\mu\left(M_{\delta, r}\left(u_{2}\right)\right)}{\mu([-r, r])}=0 .
$$

Hence, using (3.17), we obtain that

$$
\limsup _{r \rightarrow+\infty} \frac{1}{\mu([-r, r])} \int_{-r}^{r}\left(\int_{t}^{t+1}\|F(s)\|_{Y}^{p} d s\right)^{\frac{1}{p}} d \mu(t) \leq \varepsilon
$$

for all $\varepsilon>0$. Consequently,

$$
\lim _{r \rightarrow+\infty} \frac{1}{\mu([-r, r])} \int_{-r}^{r}\left(\int_{t}^{t+1}\|F(s)\|_{Y}^{p} d s\right)^{\frac{1}{p}} d \mu(t)=0 .
$$




\section{$4 \mu$-pseudo almost automorphic solutions for equation (1.4)}

In this section, we study the following semilinear nonautonomous evolution equations:

$$
u^{\prime}(t)=A_{\alpha-1}(t) u(t)+\tilde{f}(t, u(t)) \quad \text { for } t \in \mathbb{R} .
$$

We assume that $A(t), t \in \mathbb{R}$ are linear operators on a Banach space $X$ satisfying the conditions (2.1) and (2.2). Denote by $(U(t, s))_{t \geq s}$ the evolution family generated by $A(t), t \in \mathbb{R}$ on $X$ and by $\left(U_{\alpha-1}(t, s)\right)_{t \geq s}$ its extrapolated evolution family defined in Proposition 2.1 for each $0<\alpha \leq 1$. The function $\tilde{f}: \mathbb{R} \times X_{\beta}^{t} \longrightarrow$ $X_{\alpha-1}^{t}$ is locally integragble with respect to the first variable and Lipschitzian with respect to the second one.

Firstly, we prove the existence of a $\mu$-pseudo almost automorphic solution to the following ihomogeneous linear equation associated to (4.1):

$$
u^{\prime}(t)=A_{\alpha-1}(t) u(t)+h(t) \quad \text { for } t \in \mathbb{R} .
$$

Definition 4.1 A continuous function $u: \mathbb{R} \longrightarrow X_{\beta}^{t}$ is called a mild solution of equation (4.2) if it satisfies the following variation of constants formula:

$$
u(t)=U(t, \sigma) u(\sigma)+\int_{\sigma}^{t} U_{\alpha-1}(t, s) h(s) d s \quad \text { for all } t \geq \sigma,
$$

where $h: \mathbb{R} \longrightarrow X_{\beta}^{t}$ is locally integrable.

Hence, we list the following hypotheses:

(H1) The operators $A(t), t \in \mathbb{R}$ satisfy the assumptions (2.1) and (2.2).

(H2) the evolution family $(U(t, s))_{t \geq s}$ generated by $A(t), t \in \mathbb{R}$ has an exponential dichotomy on $\mathbb{R}$ with constants $N, \delta>0$, projections $P(t), t \in \mathbb{R}$, and Green's function $\Gamma$.

(H3) For all $1 \leq p<\infty$, the function $R(\omega, A(\cdot)) \in A A S^{p}(\mathbb{R}, \mathcal{L}(X))$.

(H4) There exist $0 \leq \alpha<\beta<1$ such that $X_{\alpha}^{t}=X_{\alpha}$ and $X_{\beta}^{t}=X_{\beta}$ for every $t \in \mathbb{R}$ with uniformly equivalent norms.

(H5) For all $\tau \in \mathbb{R}$, there exist $\beta>0$ and a bounded interval $I$ such that

$$
\mu(\{a+\tau: a \in A\}) \leq \beta \mu(A), \quad \text { where } A \in \mathcal{B}(\mathbb{R}) \text { and } A \cap I=\varnothing .
$$

Now, we introduce some preliminary results. Let us define the Yosida approximations $A_{n}(t)=n A(t) R(n, A(t))$ of $A(t)$ for $n>\omega$ and $t \in \mathbb{R}$. For each $n$, the operator $A_{n}$ generates an evolution family $\left(U_{n}(t, s)\right)_{t \geq s}$ on $X$. It has been shown in [24, Lemma 3.1, Proposition 3.3, Corollary 3.4] that assumptions (H1) and (H2) are satisfied by $A_{n}$ with the same constants for every $n \geq n_{0}$ (with $n_{0}>\omega$ ).

Lemma 4.1 Let (H1) and (H3) be satisfied. Then, there exists $n_{1} \geq n_{0}$ such that

$$
R\left(\omega, A_{n}(\cdot)\right) \in A A S^{p}(\mathbb{R}, \mathcal{L}(X)) \text { for all } n \geq n_{1} .
$$

Proof. Let $\left(\sigma_{l}\right)_{l \geq 0}$ be a sequence of real numbers, since $R(\omega, A(\cdot)) \in A A S^{p}(\mathbb{R}, \mathcal{L}(X))$, there exists a subsequence $\left(s_{l}\right)_{l \geq 0}$ such that

$$
\left(\int_{t}^{t+1}\left\|R\left(\omega, A\left(s+s_{l}-s_{k}\right)\right)-R(\omega, A(s))\right\|^{p} d s\right)^{\frac{1}{p}} \rightarrow 0 \text { as } k, l \rightarrow \infty
$$

for all $t \in \mathbb{R}$. Let $t \in \mathbb{R}$ and $s \in[t, t+1]$. If $n \geq n_{0}$ and $|\arg (\lambda-\omega)| \leq \theta$, then

$$
\begin{aligned}
& R\left(\omega, A_{n}\left(s+s_{l}-s_{k}\right)\right)-R\left(\omega, A_{n}(s)\right) \\
& =\frac{n^{2}}{(\omega+n)^{2}}\left(R\left(\frac{\omega n}{\omega+n}, A\left(s+s_{l}-s_{k}\right)\right)-R\left(\frac{\omega n}{\omega+n}, A(s)\right)\right) \\
& =\frac{n^{2}}{(\omega+n)^{2}} R\left(\omega, A\left(s+s_{l}-s_{k}\right)\right)\left[I-\frac{\omega^{2}}{\omega+n} R\left(\omega, A\left(s+s_{l}-s_{k}\right)\right)\right]^{-1} \\
& -\frac{n^{2}}{(\omega+n)^{2}} R(\omega, A(s))\left[I-\frac{\omega^{2}}{\omega+n} R(\omega, A(s))\right]^{-1} .
\end{aligned}
$$


Otherwise, we have

$$
\left\|\frac{\omega^{2}}{(\omega+n)^{2}} R(\omega, A(s))\right\| \leq \frac{\omega L}{(\omega+n)^{2}} \leq \frac{\omega L}{n} \leq \frac{1}{2} \text { for } n \geq n_{1}=\max \left\{n_{0}, 2 \omega L\right\} .
$$

Therefore,

$$
\left\|\left[I-\frac{\omega^{2}}{\omega+n} R(\omega, A(s))\right]^{-1}\right\| \leq 2 .
$$

Hence, from (4.5), we obtain that

$$
\begin{aligned}
\left\|R\left(\omega, A_{n}\left(s+s_{l}-s_{k}\right)\right)-R\left(\omega, A_{n}(s)\right)\right\| & \leq 2\left\|\left(R\left(\omega, A\left(s+s_{l}-s_{k}\right)\right)-R(\omega, A(s))\right)\right\| \\
& +\frac{K}{1+\omega}\left\|\left[I-\frac{\omega^{2}}{\omega+n} R\left(\omega, A\left(s+s_{l}-s_{k}\right)\right)\right]^{-1}-\left[I-\frac{\omega^{2}}{\omega+n} R(\omega, A(s))\right]^{-1}\right\| .
\end{aligned}
$$

Now, using (4.6), we obtain that

$$
\begin{aligned}
& \left\|\left[I-\frac{\omega^{2}}{\omega+n} R\left(\omega, A\left(s+s_{l}-s_{k}\right)\right)\right]^{-1}-\left[I-\frac{\omega^{2}}{\omega+n} R(\omega, A(s))\right]^{-1}\right\| \\
& \leq 4\left\|\left[I-\frac{\omega^{2}}{\omega+n} R\left(\omega, A\left(s+s_{l}-s_{k}\right)\right)\right]-\left[I-\frac{\omega^{2}}{\omega+n} R(\omega, A(s))\right]\right\| \\
& \leq 4 \omega\left\|\left(R\left(\omega, A\left(s+s_{l}-s_{k}\right)\right)-R(\omega, A(s))\right)\right\| .
\end{aligned}
$$

Consequently,

$\left\|\left(R\left(\omega, A_{n}\left(s+s_{l}-s_{k}\right)\right)-R\left(\omega, A_{n}(s)\right)\right)\right\| \leq(2+4 K)\left\|\left(R\left(\omega, A\left(s+s_{l}-s_{k}\right)\right)-R(\omega, A(s))\right)\right\| \quad$ for $n \geq n_{1} .(4.7)$

By integrating in both sides of (4.7) from $t$ to $t+1$, we obtain by (4.9) that

$$
\left(\int_{t}^{t+1}\left\|R\left(\omega, A_{n}\left(s+s_{l}-s_{k}\right)\right)-R\left(\omega, A_{n}(s)\right)\right\|^{p} d s\right)^{\frac{1}{p}} \rightarrow 0 \text { as } k, l \rightarrow \infty
$$

for all $t \in \mathbb{R}$.

Lemma 4.2 Assume that (H1)- (H3) are satisfied. Then, for every sequence $\left(\sigma_{l}\right)_{l \geq 0}$ of real numbers, there exists a subsequence $\left(s_{l}\right)_{l \geq 0}$ such that for every $\eta>0$ and $t, s \in \mathbb{R}$, there exists $l(\eta, t, s)>0$ satisfying

$$
\left\|\Gamma_{n}\left(t+s_{l}-s_{k}, s+s_{l}-s_{k}\right)-\Gamma_{n}(t, s)\right\| \leq c n^{2} \eta
$$

for a large $n$ and $l, k \geq l(\eta, t, s)$.

Proof. Let $\left(\sigma_{l}\right)_{l \geq 0}$ be a sequence of real numbers. Since $R(\omega, A(\cdot)) \in A A S^{p}(\mathbb{R}, \mathcal{L}(X))$ there exists a subsequence $\left(s_{l}\right)_{l \geq 0}$ such that

$$
\left(\int_{t}^{t+1}\left\|R\left(\omega, A\left(s+s_{l}-s_{k}\right)\right)-R(\omega, A(s))\right\|^{p} d s\right)^{\frac{1}{p}} \rightarrow 0 \text { as } k, l \rightarrow \infty
$$

for all $t \in \mathbb{R}$. Let $t, s \in \mathbb{R}$. Arguing as in [24], we obtain that

$$
\begin{aligned}
\Gamma_{n}\left(t+s_{l}-s_{k}, s+s_{l}-s_{k}\right)-\Gamma_{n}(t, s)= & \int_{\mathbb{R}} \Gamma_{n}(t, \sigma)\left(A_{n}(\sigma)-\omega\right)\left[R\left(\omega, A_{n}\left(\sigma+s_{l}-s_{k}\right)\right)-R\left(\omega, A_{n}(\sigma)\right)\right] \\
& \times\left(A_{n}\left(\sigma+s_{l}-s_{k}\right)-\omega\right) \Gamma_{n}\left(\sigma+s_{l}-s_{k}, s+s_{l}-s_{k}\right) d \sigma
\end{aligned}
$$


for $l, k \in \mathbb{N}$ and large $n$. From (4.10), (4.7), [24, Corollary 3.4] and Hölder inequality, we claim that

$$
\begin{aligned}
& \left\|\Gamma_{n}\left(t+s_{l}-s_{k}, s+s_{l}-s_{k}\right)-\Gamma_{n}(t, s)\right\| \\
\leq & c n^{2} \int_{\mathbb{R}} e^{-\frac{3}{4 \delta}|t-\sigma|} e^{-\frac{3}{4 \delta}|s-\sigma|}\left\|R\left(\omega, A_{n}\left(\sigma+s_{l}-s_{k}\right)\right)-R\left(\omega, A_{n}(\sigma)\right)\right\| d \sigma \\
\leq & c n^{2}(2+4 K) \int_{\mathbb{R}} e^{-\frac{3}{4 \delta}|t-\sigma|} e^{-\frac{3}{4 \delta}|s-\sigma|}\left[R\left(\omega, A\left(\sigma+s_{l}-s_{k}\right)\right)-R(\omega, A(\sigma))\right] d \sigma \\
\leq & c n^{2}(2+4 K)\left(\int_{\mathbb{R}} e^{-\frac{3 q}{4 \delta}|t-\sigma|} d \sigma\right)^{\frac{1}{q}}\left(\int_{\mathbb{R}} e^{-\frac{3 p}{4 \delta}|s-\sigma|}\left\|R\left(\omega, A\left(\sigma+s_{l}-s_{k}\right)\right)-R(\omega, A(\sigma))\right\|^{p} d \sigma\right)^{\frac{1}{p}} \\
= & c n^{2}(2+4 K)\left(\frac{3 q}{2 \delta}\right)^{\frac{1}{q}} \underbrace{\left(\int_{\mathbb{R}} e^{-\frac{3 p}{4 \delta}|s-\sigma|}\left\|R\left(\omega, A\left(\sigma+s_{l}-s_{k}\right)\right)-R(\omega, A(\sigma))\right\|^{p} d \sigma\right)^{\frac{1}{p}}}_{\text {(I) }}
\end{aligned}
$$

we develop the formula (I), we obtain that

$$
\begin{aligned}
\int_{\mathbb{R}} e^{-\frac{3 p}{4 \delta}|s-\sigma|}\left\|R\left(\omega, A\left(\sigma+s_{l}-s_{k}\right)\right)-R(\omega, A(\sigma))\right\|^{p} d \sigma= & \int_{-\infty}^{s} e^{-\frac{3 p}{4 \delta}(s-\sigma)}\left\|R\left(\omega, A\left(\sigma+s_{l}-s_{k}\right)\right)-R(\omega, A(\sigma))\right\|^{p} d \sigma \\
& +\int_{s}^{+\infty} e^{\frac{3 p}{4 \delta}(s-\sigma)}\left\|R\left(\omega, A\left(\sigma+s_{l}-s_{k}\right)\right)-R(\omega, A(\sigma))\right\|^{p} d \sigma \\
:= & I_{1}+I_{2} .
\end{aligned}
$$

Using (4.9) and the Lebesgue's Dominated Convergence Theorem, we get that

$$
\begin{aligned}
I_{1} & =\sum_{m=1}^{\infty} \int_{s-m}^{s-m+1} e^{-\frac{3 p}{4 \delta}(s-\sigma)}\left\|R\left(\omega, A\left(\sigma+s_{l}-s_{k}\right)\right)-R(\omega, A(\sigma))\right\|^{p} d \sigma \\
& \leq \sum_{m=1}^{\infty} e^{-\frac{3 p}{4 \delta}(m-1)} \int_{s-m}^{s-m+1}\left\|R\left(\omega, A\left(\sigma+s_{l}-s_{k}\right)\right)-R(\omega, A(\sigma))\right\|^{p} d \sigma \rightarrow 0 \text { as } k, l \rightarrow \infty,
\end{aligned}
$$

and

$$
\begin{aligned}
I_{2} & =\sum_{m=0}^{\infty} \int_{s+m}^{s+m+1} e^{-\frac{3 p}{4 \delta}(s-\sigma)}\left\|R\left(\omega, A\left(\sigma+s_{l}-s_{k}\right)\right)-R(\omega, A(\sigma))\right\|^{p} d \sigma \\
& \leq \sum_{m=0}^{\infty} e^{-\frac{3 p}{4 \delta} m} \int_{s+m}^{s+m+1}\left\|R\left(\omega, A\left(\sigma+s_{l}-s_{k}\right)\right)-R(\omega, A(\sigma))\right\|^{p} d \sigma \rightarrow 0 \text { as } k, l \rightarrow \infty .
\end{aligned}
$$

Hence, for every $\eta>0$, there exists $l(\eta, t, s)>0$ such that

$$
\left\|\Gamma_{n}\left(t+s_{l}-s_{k}, s+s_{l}-s_{k}\right)-\Gamma_{n}(t, s)\right\| \leq c n^{2} \eta
$$

for a large $n$ and $l, k \geq l(\eta, t, s)$.

In the next Theorem, we show that, to have $\Gamma$ almost automorphic, we only need that $R(\omega, A(\cdot))$ to be $S^{p}$-almost automorphic.

Theorem 4.1 Let (H1)- (H3) be satisfied. Then, $\Gamma \in b A A(\mathbb{R}, X)$.

Proof. It suffices to use Lemma 4.2 and arguing as the same as in the proof of [10, Proposition 3.3 ].

Now, we prove the existence of a unique $\mu$-pseudo almost automorphic mild solution to (4.2).

Theorem 4.2 Let $1 \leq p<\infty, 0 \leq \beta<\alpha \leq 1$ and $h \in B S^{p}\left(\mathbb{R}, X_{\alpha-1}\right)$. Assume that (H1)-(H4) are satisfied. Then, the following hold:

(i) The equation (4.2) has a unique bounded mild solution $u: \mathbb{R} \longrightarrow X_{\beta}$ given by

$$
u(t)=\int_{\mathbb{R}} \Gamma_{\alpha-1}(t, s) h(s) d s \quad \text { for all } t \in \mathbb{R} .
$$


(ii) If $h \in A A S^{p}\left(\mathbb{R}, X_{\alpha-1}\right)$, then $u \in A A\left(\mathbb{R}, X_{\beta}\right)$.

Proof. Let $1 \leq p<\infty, 0 \leq \beta<\alpha \leq 1$ and $h \in B S^{p}\left(\mathbb{R}, X_{\alpha-1}\right)$.

(i) Firstly, let us prove that formula (4.16) is well-defined. Indeed, we take

$$
u^{s}(t):=\int_{-\infty}^{t} U_{\alpha-1}(t, s) P_{\alpha-1}(s) h(s) d s \quad \text { and } \quad u^{u}(t):=-\int_{t}^{+\infty} \tilde{U}_{\alpha-1}(t, s) Q_{\alpha-1}(t) h(s) d s \text { for all } t \in \mathbb{R} \text {. }
$$

Using Theorem 2.3 and Hölder's inequality, we obtain that

$$
\begin{aligned}
\left\|u^{s}(t)\right\|_{\beta} & \leq \int_{-\infty}^{t}\left\|U_{\alpha-1}(t, s) P_{\alpha-1}(s) h(s)\right\|_{\beta} d s \\
& \leq m(\alpha, \beta) \int_{-\infty}^{t} e^{-\gamma(t-s)}(t-s)^{\alpha-\beta-1}\|h(s)\|_{\alpha-1} d s \\
& \leq m(\alpha, \beta)\left[\int_{-\infty}^{t} e^{-\frac{q \gamma(t-s)}{2}}(t-s)^{q(\alpha-\beta-1)} d s\right]^{\frac{1}{q}}\left[\int_{-\infty}^{t} e^{-\frac{p \gamma(t-s)}{2}}\|h(s)\|_{\alpha-1}^{p} d s\right]^{\frac{1}{p}} \\
& =m(\alpha, \beta)\left(\frac{2}{q \gamma}\right)^{(\alpha-\beta-1)}[q(\alpha-\beta-1) \Gamma(q(\alpha-\beta-1))]^{\frac{1}{q}}\left[\int_{-\infty}^{t} e^{-\frac{p \gamma(t-s)}{2}}\|h(s)\|_{\alpha-1}^{p} d s\right]^{\frac{1}{p}} \\
& =m(\alpha, \beta)\left(\frac{2}{q \gamma}\right)^{(\alpha-\beta-1)}[q(\alpha-\beta-1) \Gamma(q(\alpha-\beta-1))]^{\frac{1}{q}}\left[\sum_{k=1}^{\infty} \int_{t-k}^{t-k+1} e^{-\frac{p \gamma(t-s)}{2}}\|h(s)\|_{\alpha-1}^{p} d s\right]^{\frac{1}{p}} \\
& \leq m(\alpha, \beta)\left(\frac{2}{q \gamma}\right)^{(\alpha-\beta-1)}[q(\alpha-\beta-1) \Gamma(q(\alpha-\beta-1))]^{\frac{1}{q}}[\Gamma(q(\alpha-\beta))]^{\frac{1}{q}}\left[\sum_{k=1}^{\infty} e^{-\frac{p \gamma(k-1)}{2}} \int_{t-k}^{t-k+1}\|h(s)\|_{\alpha-1}^{p} d s\right]^{\frac{1}{p}} \\
& \leq m(\alpha, \beta)\left(\frac{2}{q \gamma}\right)^{(\alpha-\beta-1)}[q(\alpha-\beta-1) \Gamma(q(\alpha-\beta-1))]^{\frac{1}{q}}\left[\frac{1}{1-e^{-\frac{p \gamma}{2}}}\right]^{\frac{1}{p}}\|h\|_{B S_{\alpha-1}^{p}} \text { for all } t \in \mathbb{R} .
\end{aligned}
$$

Arguing as above, we obtain that

$$
\begin{aligned}
\left\|u^{u}(t)\right\|_{\beta} & \leq \int_{t}^{+\infty}\left\|\tilde{U}_{\alpha-1}(t-s) Q_{\alpha-1}(t) h(s)\right\| d s \\
& \leq c(\alpha, \beta) \int_{t}^{+\infty} e^{\delta(t-s)}\|h(s)\|_{\alpha-1} d s \\
& \leq c(\alpha, \beta)\left[\int_{t}^{+\infty} e^{\frac{q \delta(t-s)}{2}} d s\right]^{\frac{1}{q}}\left[\int_{t}^{+\infty} e^{\frac{p \delta(t-s)}{2}}\|h(s)\|_{\alpha-1}^{p} d s\right]^{\frac{1}{p}} \\
& \leq c(\alpha, \beta)\left(\frac{2}{q \delta}\right)^{\frac{1}{q}}\left[\frac{1}{1-e^{-\frac{p \delta}{2}}}\right]^{\frac{1}{p}}\|h\|_{B S_{\alpha-1}^{p}} \text { for all } t \in \mathbb{R} .
\end{aligned}
$$

Thus, the integrals given by $u^{s}$ and $u^{u}$ are both defined. Moreover,

$$
\sup _{t \in \mathbb{R}}\|u(t)\|_{\beta}<\infty,
$$

which proves that $u$ is bounded.

Now, we show that $u$ defined by (4.3) is the unique mild solution of (4.2). In fact, let $\tilde{u}$ be the mild solution of the equation (4.2) given by (4.3). Then, by using the uniqueness of the decomposition of $X_{\beta}^{t}$, we claim that $\tilde{u}=\tilde{u}^{s}+\tilde{u}^{u}$, where

$$
\tilde{u}^{s}(t):=U_{\alpha-1}(t, \sigma) P(\sigma) u(\sigma)+\int_{\sigma}^{t} U_{\alpha-1}(t, s) P_{\alpha-1}(s) h(s) d s \quad \text { for all } t \geq \sigma
$$

and

$$
\tilde{u}^{u}(t):=\tilde{U}_{\alpha-1}(t, \sigma) Q_{\alpha-1}(t) u(\sigma)+\int_{\sigma}^{t} \tilde{U}_{\alpha-1}(t, s) Q_{\alpha-1}(t) h(s) d s \quad \text { for all } t, \sigma \in \mathbb{R} .
$$


From the estimates given in Proposition 2.3, (4.33) and (4.33) we claim that $\tilde{u}^{s}$ and $\tilde{u}^{u}$ are bounded respectively and by letting $\sigma \rightarrow-\infty$ in (4.20) and $\sigma \rightarrow+\infty$ in (4.21) respectively, we obtain that

$$
\tilde{u}^{s}(t):=\int_{-\infty}^{t} U_{\alpha-1}(t, s) P_{\alpha-1}(s) h(s) d s \text { for all } t \in \mathbb{R},
$$

and

$$
\tilde{u}^{u}(t):=-\int_{t}^{+\infty} \tilde{U}_{\alpha-1}(t, s) Q_{\alpha-1}(t) h(s) d s \text { for all } t \in \mathbb{R} .
$$

Therefore, we have $u(t)=\tilde{u}(t)$ for all $t \in \mathbb{R}$, which prove the uniqueness.

(ii) Let $h \in A A S^{p}\left(\mathbb{R}, X_{\alpha-1}\right)$. For each $k \in \mathbb{N}$, we define:

$$
\Phi_{k}(t):=\int_{t-k-1}^{t-k} U_{\alpha-1}(t, \sigma) P_{\alpha-1}(\sigma) h(\sigma) d \sigma \quad \text { for } t \in \mathbb{R}
$$

and

$$
\Psi_{k}(t):=\int_{t-k-1}^{t-k} \tilde{U}_{\alpha-1}(t, \sigma) Q_{\alpha-1}(t) h(\sigma) d \sigma \quad \text { for } t \in \mathbb{R} .
$$

Let $k \in \mathbb{N}$. Then, we have

$$
\begin{aligned}
\left\|\Phi_{k}(t)\right\|_{\beta} & \leq \int_{t-k-1}^{t-k}\left\|U_{\alpha-1}(t, s) P_{\alpha-1}(s) h(s)\right\| d s \\
& \leq m(\alpha, \beta) \int_{t-k-1}^{t-k} e^{-\gamma(t-s)}(t-s)^{\alpha-\beta-1}\|h(s)\|_{\alpha-1} d s \\
& \leq m(\alpha, \beta)\left[\int_{t-k-1}^{t-k} e^{\frac{-q \gamma(t-s)}{2}}(t-s)^{q(\alpha-\beta-1)} d s\right]^{\frac{1}{q}}\left[\int_{t-k-1}^{t-k} e^{\frac{-p \gamma(t-s)}{2}}\|h(s)\|_{\alpha-1}^{p} d s\right]^{\frac{1}{p}} \\
& \leq m(\alpha, \beta)\left(\frac{q \gamma}{2}\right)^{(1-\alpha+\beta)}\left[\int_{0}^{+\infty} e^{-s} s^{q(\alpha-\beta-1)} d s\right]^{\frac{1}{q}} e^{\frac{-\gamma k}{2}}\|h\|_{B S_{\alpha-1}^{p}} \\
& =m(\alpha, \beta)\left(\frac{q \gamma}{2}\right)^{(1-\alpha+\beta)}[q(\alpha-\beta-1) \Gamma(q(\alpha-\beta-1))]^{\frac{1}{q}}\|h\|_{B S_{\alpha-1}^{p}} e^{\frac{-\gamma k}{2}} \quad \text { for all } t \in \mathbb{R}
\end{aligned}
$$

and

$$
\begin{aligned}
\left\|\Psi_{k}(t)\right\|_{\beta} & \leq \int_{t+k}^{t+k+1}\left\|\tilde{U}_{\alpha-1}(t, s) Q_{\alpha-1}(t) h(s)\right\| d s \\
& \leq c(\alpha, \beta) \int_{t+k}^{t+k+1} e^{\delta(t-s)}\|h(s)\|_{\alpha-1} d s \\
& \leq c(\alpha, \beta)\left[\int_{t+k}^{t+k+1} e^{\frac{q \delta(t-s)}{2}} d s\right]^{\frac{1}{q}}\left[\int_{t+k}^{t+k+1} e^{\frac{p \delta(t-s)}{2}}\|h(s)\|_{\alpha-1}^{p} d s\right]^{\frac{1}{p}} \\
& \leq c(\alpha, \beta)\left[\int_{0}^{+\infty} e^{\frac{-q \delta s}{2}} d s\right]^{\frac{1}{q}}\|h\|_{B S_{\alpha-1}^{p}} \\
& =c(\alpha, \beta)\left(\frac{q \delta}{2}\right)^{-\frac{1}{q}}\|h\|_{B S_{\alpha-1}^{p}} e^{\frac{-\delta k}{2}} \text { for all } t \in \mathbb{R} .
\end{aligned}
$$

Since $\sum_{k \geq 0} e^{\frac{-\xi k}{2}}=\frac{1}{1-e^{\frac{-\xi}{2}}}<\infty$, for $\xi:=\gamma, \delta$, it follows from Weierstrass Theorem that the series $\sum_{k \geq 0} \Phi_{k}(t)$ and $\sum_{k \geq 0} \Psi_{k}(t)$ are uniformly convergent on $\mathbb{R}$. Then, we define

$$
\Phi(t):=\sum_{k \geq 0} \Phi_{k}(t) \quad \text { and } \quad \Psi(t):=\sum_{k \geq 0} \Psi_{k}(t) \quad \text { for all } t \in \mathbb{R}
$$


In addition, we have

$$
u(t)=\Phi(t)+\Psi(t) \quad \text { for all } t \in \mathbb{R},
$$

where $\Phi(t)=\int_{-\infty}^{t} U_{\alpha-1}(t, s) P_{\alpha-1}(s) h(s) d s \quad$ and $\quad \Psi(t)=\int_{t}^{+\infty} \tilde{U}_{\alpha-1}(t, s) Q_{\alpha-1}(t) h(s) d s \quad$ for $t \in \mathbb{R}$. In fact, let $n \in \mathbb{N}$. Then,

$$
\begin{aligned}
\left\|\Phi(t)-\sum_{k=0}^{n} \Phi_{k}(t)\right\| & =\left\|\int_{-\infty}^{t} U_{\alpha-1}(t, s) P_{\alpha-1}(s) h(s) d s-\sum_{k \geq 0} \int_{t-k-1}^{t-k} U_{\alpha-1}(t, \sigma) P_{\alpha-1}(\sigma) h(\sigma) d \sigma\right\| \\
& \leq \sum_{k \geq n+1} \int_{t-k-1}^{t-k}\left\|U_{\alpha-1}(t, \sigma) P_{\alpha-1}(\sigma) h(\sigma)\right\| d \sigma \\
& \leq m(\alpha, \beta)\left(\frac{q \gamma}{2}\right)^{(1-\alpha+\beta)}[q(\alpha-\beta-1) \Gamma(q(\alpha-\beta-1))]^{\frac{1}{q}}\|h\|_{B S_{\alpha-1}^{p}} \sum_{k \geq n+1} e^{\frac{-\gamma k}{2}} \rightarrow 0 \quad \text { as } n \rightarrow \infty .
\end{aligned}
$$

uniformly in $t \in \mathbb{R}$.

By the same way, we prove the result for $\Psi$. To conclude, it suffices to prove that for each $k \in \mathbb{N}$, $\Phi_{k}$ and $\Psi_{k}$ belong to $A A\left(\mathbb{R}, X_{\beta}\right)$. Let $\left(\sigma_{n}\right)_{n} \subset \mathbb{R}$ be a sequence of real numbers. Since $h \in A A S^{p}\left(\mathbb{R}, X_{\alpha-1}\right)$, and $\Gamma \in b A A(\mathbb{R}, X)$ it follows that:

(A) there exist a subsequence $\left(s_{n}\right)_{n} \subset\left(\sigma_{n}\right)_{n}$ and a measurable function $\tilde{g}: \mathbb{R} \longrightarrow X_{\alpha-1}$ such that

$$
\lim _{n} \int_{t}^{t+1}\left(\left\|g\left(s+s_{n}\right)-\tilde{g}(s)\right\|_{\alpha-1}^{p} d s\right)^{\frac{1}{p}}=0 \text { and } \lim _{n} \int_{t}^{t+1}\left(\left\|\tilde{g}\left(s-s_{n}\right)-g(s)\right\|_{\alpha-1}^{p} d s\right)^{\frac{1}{p}}=0
$$

for all $t \in \mathbb{R}$.

(B) there exist a subsequence $\left(s_{n}^{\prime}\right)_{n} \subset\left(\sigma_{n}\right)_{n}$ and a measurable function $\tilde{\Gamma}: \mathbb{R}^{2} \longrightarrow X$ such that

$$
\lim _{n}\left\|\Gamma\left(t+s_{n}^{\prime}, s+s_{n}^{\prime}\right) x-\tilde{\Gamma}(t, s) x\right\|=0 \text { and } \lim _{n}\left\|\tilde{\Gamma}\left(t-s_{n}^{\prime}, s-s_{n}^{\prime}\right) x-\Gamma(t, s) x\right\|=0
$$

for all $t, s \in \mathbb{R}$ and $x \in X$.

Let $\left(\sigma_{n}^{\prime \prime}\right)_{n} \subset\left(\sigma_{n}^{\prime}\right)_{n},\left(\sigma_{n}\right)_{n}$ be a subsequence of the sequences $\left(\sigma_{n}^{\prime}\right)_{n}$ and $\left(\sigma_{n}\right)_{n}$ respectively. Then, for each $k \in \mathbb{N}$, we have

$$
\begin{aligned}
& \left\|\Phi_{k}\left(t+\sigma_{n}^{\prime \prime}\right)-\int_{t-k-1}^{t-k} \tilde{\Gamma}_{\alpha-1}(t, s) \tilde{h}(s) d s\right\| \\
= & \left\|\int_{k}^{k+1}\left[\Gamma_{\alpha-1}\left(t+\sigma_{n}^{\prime \prime}, t+\sigma_{n}^{\prime \prime}-s\right) h\left(t+\sigma_{n}^{\prime \prime}-s\right) d s-\tilde{\Gamma}_{\alpha-1}(t, t-s) \tilde{h}(t-s)\right] d s\right\| \\
\leq & \int_{k}^{k+1}\left\|\left[\Gamma_{\alpha-1}\left(t+\sigma_{n}^{\prime \prime}, t+\sigma_{n}^{\prime \prime}-s\right)-\tilde{\Gamma}_{\alpha-1}(t, t-s)\right] \tilde{h}(t-s)\right\|_{\alpha-1} d s \\
+ & \int_{k}^{k+1}\left\|\Gamma_{\alpha-1}\left(t+\sigma_{n}^{\prime \prime}, t+\sigma_{n}^{\prime \prime}-s\right)\left[h\left(t+\sigma_{n}^{\prime \prime}-s\right)-\tilde{h}(t-s)\right]\right\|_{\alpha-1} d s \\
\leq & \int_{k}^{k+1}\left\|\left[\Gamma_{\alpha-1}\left(t+\sigma_{n}^{\prime \prime}, t+\sigma_{n}^{\prime \prime}-s\right)-\tilde{\Gamma}_{\alpha-1}(t, t-s)\right] \tilde{h}(t-s)\right\|_{\alpha-1} d s \\
+ & m(\alpha, \beta)\left[\int_{k}^{k+1} e^{-q \gamma s} s^{q(\alpha-\beta-\tilde{\varepsilon}-1)} d s\right]^{\frac{1}{q}}\left[\int_{k}^{k+1}\left\|h\left(t+\sigma_{n}^{\prime \prime}-s\right)-\tilde{h}(t-s)\right\|_{\alpha-1}^{p} d s\right]^{\frac{1}{p}} \\
\leq & \int_{k}^{k+1}\left\|\left[\Gamma_{\alpha-1}\left(t+\sigma_{n}^{\prime \prime}, t+\sigma_{n}^{\prime \prime}-s\right)-\tilde{\Gamma}_{\alpha-1}(t, t-s)\right] \tilde{h}(t-s)\right\|_{\alpha-1} d s \\
+ & m(\alpha, \beta)(q \gamma)^{(1-\alpha+\beta)}[q(\alpha-\beta-1) \Gamma(q(\alpha-\beta-1))]^{\frac{1}{q}}\left[\int_{k}^{k+1}\left\|h\left(t+\sigma_{n}^{\prime \prime}-s\right)-\tilde{h}(t-s)\right\|_{\alpha-1}^{p} d s\right]^{\frac{1}{p}} \\
:= & J_{1}+J_{2} .
\end{aligned}
$$

By using (A), $J_{2} \rightarrow 0$ as $n \rightarrow \infty$ for all $t \in \mathbb{R}$. From (B) and since

$$
\left\|\left[\Gamma_{\alpha-1}\left(t+\sigma_{n}^{\prime \prime}, t+\sigma_{n}^{\prime \prime}-s\right)-\tilde{\Gamma}_{\alpha-1}(t, t-s)\right] \tilde{h}(t-s)\right\|_{\alpha-1} \leq 2 m(\alpha, \beta) e^{-\gamma s} s^{\alpha-\beta-1}\|\tilde{h}(t-s)\|_{\alpha-1}
$$


it follows in view of the dominated convergence Theorem, that $J_{1} \rightarrow 0$ as $n \rightarrow \infty$ for all $t \in \mathbb{R}$. Hence

$$
\lim _{n}\left\|\Phi_{k}\left(t+\sigma_{n}^{\prime \prime}\right)-\int_{t-k-1}^{t-k} \tilde{\Gamma}_{\alpha-1}(t, s) \tilde{h}(s) d s\right\|=0 \quad \text { for all } t \in \mathbb{R} .
$$

Analogously, we prove that

$$
\lim _{n}\left\|\int_{k}^{k+1} \tilde{\Gamma}_{\alpha-1}\left(t-\sigma_{n}^{\prime \prime}, t-\sigma_{n}^{\prime \prime}-s\right) \tilde{h}\left(t-\sigma_{n}^{\prime \prime}-s\right) d s-\Phi_{k}(t)\right\|=0 \quad \text { for all } t \in \mathbb{R} .
$$

Therefore, for each $k \in \mathbb{N}, \Phi_{k} \in A A\left(\mathbb{R}, X_{\beta}\right)$. We recall that the series $\sum_{k \geq 0} \Phi_{k}(t)$ is uniformly convergent on $\mathbb{R}$, which implies that $\Phi \in A A\left(\mathbb{R}, X_{\beta}\right)$.

Similarly, we prove that $\Psi \in A A\left(\mathbb{R}, X_{\beta}\right)$.

Consequently, $u \in A A\left(\mathbb{R}, X_{\beta}\right)$.

In the next Theorem, we show that to have a $\mu$-pseudo almost automorphic mild solution, we only need $h$ to be $\mu$-pseudo almost automorphic in the sense of Stepanov. To get that purpose, we need the following Lemma.

Lemma 4.3 Let $\mu \in \mathcal{M}$ and $f \in \mathcal{E}^{p}(\mathbb{R}, X, \mu)$. Then,

$$
\frac{1}{\mu([-r, r])} \int_{[-r, r]} \int_{[t, t+1]}\|f(s)\|^{p} d s d \mu(t) \rightarrow 0 \quad \text { as } r \rightarrow \infty .
$$

Proof. Let $f \in \mathcal{E}^{p}(\mathbb{R}, X, \mu)$. Then, for every $r>0$

$$
\frac{1}{\mu([-r, r])} \int_{[-r, r]} \int_{[t, t+1]}\|f(s)\|^{p} d s d \mu(t) \leq\|f\|_{B S^{p}}^{p-1} \frac{1}{\mu([-r, r])} \int_{[-r, r]}\left(\int_{[t, t+1]}\|f(s)\|^{p} d s\right)^{\frac{1}{p}} d \mu(t) .
$$

By the fact that $f \in \mathcal{E}^{p}(\mathbb{R}, X, \mu)$, the right hand side in (4.27) $\rightarrow 0$ as $r \rightarrow \infty$. Hence, (4.26) holds.

Theorem 4.3 Let $\mu \in \mathcal{M}$ and $0 \leq \beta<\alpha \leq 1$. Assume that (H1)-(H5) are satisfied and that $h \in$ $P A A S^{p}\left(\mathbb{R}, X_{\alpha-1}, \mu\right)$. Then, equation (4.2) has a unique mild solution $u \in P A A\left(\mathbb{R}, X_{\beta}, \mu\right)$ given by the formula (4.16).

Proof. Let $h:=\tilde{h}+\varphi \in \operatorname{PAPS}^{p}\left(\mathbb{R}, X_{\alpha-1}, \mu\right)$ where $\tilde{h} \in A A S^{p}\left(\mathbb{R}, X_{\alpha-1}\right)$ and $\varphi \in \mathcal{E}^{p}\left(\mathbb{R}, X_{\alpha-1}, \mu\right)$. Then, the mild solution of (4.2) has the unique decomposition:

$$
u=u_{1}+u_{2}
$$

where, for all $t \in \mathbb{R}$, we have

$$
u_{1}(t)=\int_{\mathbb{R}} \Gamma_{\alpha-1}(t, s) \tilde{h}(s) d s
$$

and

$$
\begin{aligned}
u_{2}(t) & =\int_{\mathbb{R}} \Gamma_{\alpha-1}(t, s) \varphi(s) d s \\
& :=u_{2}^{s}(t)+u_{2}^{u}(t)
\end{aligned}
$$

where

$$
u_{2}^{s}(t):=\int_{-\infty}^{t} U_{\alpha-1}(t, s) P_{\alpha-1}(s) \varphi(s) d s \quad \text { and } \quad u_{2}^{u}(t):=-\int_{t}^{+\infty} \tilde{U}_{\alpha-1}(t, s) Q_{\alpha-1}(t) \varphi(s) d s .
$$


Using Theorem 4.2, we obtain that $u_{1} \in A A\left(\mathbb{R}, X_{\alpha-1}\right)$. Let us prove that $u_{2} \in \mathcal{E}\left(\mathbb{R}, X_{\alpha-1}, \mu\right)$. It suffices to show that $u_{2}^{s}, u_{2}^{u} \in \mathcal{E}\left(\mathbb{R}, X_{\alpha-1}, \mu\right)$. In fact, let $r>0$, then

$$
\begin{aligned}
& \frac{1}{\mu([-r, r])} \int_{[-r, r]}\left\|u_{2}^{s}(s)\right\|_{\beta} d s d \mu(t) \\
& \leq \frac{1}{\mu([-r, r])} \int_{[-r, r]} \int_{-\infty}^{t}\left\|U_{\alpha-1}(t, s) P_{\alpha-1}(s) h_{2}(s)\right\|_{\alpha-1} d s d \mu(t) \\
& \leq \frac{m(\alpha, \beta)}{\mu([-r, r])} \int_{[-r, r]} \int_{-\infty}^{t} e^{-\gamma(t-s)}(t-s)^{(\alpha-\beta-1)}\left\|h_{2}(s)\right\|_{\alpha-1} d s d \mu(t) \\
& \leq \frac{m(\alpha, \beta)}{\mu([-r, r])} \int_{[-r, r]}\left[\int_{-\infty}^{t} e^{-q \frac{\gamma}{2}(t-s)}(t-s)^{q(\alpha-\beta-1)} d s\right]^{\frac{1}{q}}\left[\sum_{k=0}^{\infty} \int_{t-k}^{t-k+1} e^{-p \frac{\gamma}{2}(t-s)}\left\|h_{2}(s)\right\|_{\alpha-1}^{p} d s\right]^{\frac{1}{p}} d \mu(t) \\
& \leq m(\alpha, \beta)\left(\frac{q \gamma}{2}\right)^{(1-\alpha+\beta)}[q(\alpha-\beta-1) \Gamma(q(\alpha-\beta-1))]^{\frac{1}{q}} \frac{1}{\mu([-r, r])} \int_{[-r, r]}\left[\sum_{k=0}^{\infty} e^{-p \frac{\gamma}{2} k} \int_{t}^{t+1}\left\|h_{2}(s-k)\right\|^{p} d s\right]^{\frac{1}{p}} d \mu(t) \\
& \leq m(\alpha, \beta)\left(\frac{q \gamma}{2}\right)^{(1-\alpha+\beta)}[q(\alpha-\beta-1) \Gamma(q(\alpha-\beta-1))]^{\frac{1}{q}} \frac{1}{\mu\left([-r, r]^{1-\frac{1}{q}}\right.}\left[\int_{[-r, r]} \sum_{k=0}^{\infty} e^{-p \frac{\gamma}{2} k} \int_{t}^{t+1}\left\|h_{2}(s-k)\right\|^{p} d s d \mu(t)\right]^{\frac{1}{p}} \\
& =m(\alpha, \beta)\left(\frac{q \gamma}{2}\right)^{(1-\alpha+\beta)}[q(\alpha-\beta-1) \Gamma(q(\alpha-\beta-1))]^{\frac{1}{q}}[\underbrace{\sum_{k=0}^{\infty} e^{-p \frac{\gamma}{2} k} \frac{1}{\mu([-r, r]} \int_{[-r, r]} \int_{t}^{t+1}\left\|h_{2}(s-k)\right\|^{p} d s d \mu(t)}_{\text {(III) }}]^{\frac{1}{p}}
\end{aligned}
$$

Using Proposition 2.4-(iii) and Lemma 4.3, we deduce for each $k \in \mathbb{N}$, that

$$
\lim _{r \rightarrow+\infty} \frac{1}{\mu([-r, r])} \int_{[-r, r]} \int_{t}^{t+1}\left\|h_{2}(s-k)\right\|^{p} d s d \mu(t)=0 .
$$

Since,

$$
\text { (III) } \leq \sum_{k=0}^{\infty} e^{-p \frac{\gamma}{2} k}\left\|h_{2}\right\|_{B S^{p}}
$$

it holds that the series in (III) is uniformly convergent in $r$. Therefore, in view of the dominated convergence Theorem, we obtain that

$$
\lim _{r \rightarrow+\infty} \frac{1}{\mu([-r, r])} \int_{[-r, r]}\left\|u_{2}^{s}(t)\right\| d \mu(t)=0 .
$$

By the same way, we obtain that

$$
\lim _{r \rightarrow+\infty} \frac{1}{\mu([-r, r])} \int_{[-r, r]}\left\|u_{2}^{u}(t)\right\| d \mu(t)=0 .
$$

From (4.28) and (4.29), we claim that

$$
\lim _{r \rightarrow+\infty} \frac{1}{\mu([-r, r])} \int_{[-r, r]}\left\|u_{2}(t)\right\| d \mu(t)=0 .
$$

Hence, $u_{2} \in \mathcal{E}(\mathbb{R}, X, \mu)$.

Now, we return to the semilinear evolution equation (4.1). We need the following additional assumption:

(H6) For all $p \geq 1$, there exists a nonegative function $L_{\tilde{f}}(\cdot) \in B S^{p}(\mathbb{R}, \mathbb{R})$ such that

$$
\|\tilde{f}(t, x)-\tilde{f}(t, y)\|_{\alpha-1} \leq L_{\tilde{f}}(t)\|x-y\|_{\beta} \quad \text { for all } t \in \mathbb{R}, x, y \in X_{\beta} .
$$


Definition 4.2 A continuous function $u: \mathbb{R} \longrightarrow X_{\beta}$ is called a mild solution of equation (4.2) if it satisfies the following variation of constants formula:

$$
u(t)=U(t, \sigma) u(\sigma)+\int_{\sigma}^{t} U_{\alpha-1}(t, s) \tilde{f}(s, u(s)) d s \quad \text { for all } t \geq \sigma .
$$

Remark 4.1 The hypothesis (H5) implies the condition $\left(\mathbf{C}^{\prime}\right)$ in Theorem 3.3. In fact, let $B$ be a bounded subset of $X_{\beta}$, i.e., there exists $M>0$ such that

$$
\|x\|_{\beta} \leq M \text { for all } x \in B \text {. }
$$

Since $\tilde{f}$ satisfies (H5), it follows for all $x \in B$, that

$$
\begin{aligned}
\left(\int_{t}^{t+1}\|\tilde{f}(s, x)\|_{\alpha-1}^{p} d s\right)^{\frac{1}{p}} & \leq\left(\int_{t}^{t+1} L(s)^{p} d s\right)^{\frac{1}{p}}\|x\|_{\beta}+\left(\int_{t}^{t+1}\|\tilde{f}(s, 0)\|_{\alpha-1}^{p} d s\right)^{\frac{1}{p}} \\
& \leq\|L\|_{B S^{p}} M+\|\tilde{f}(\cdot, 0)\|_{B S^{p}} \quad \text { for all } t \in \mathbb{R} .
\end{aligned}
$$

Therefore,

$$
\sup _{t \in \mathbb{R}}\left(\int_{t}^{t+1}\|\tilde{f}(s, x)\|_{\alpha-1}^{p} d s\right)^{\frac{1}{p}} \leq\|L\|_{B S^{p}} M+\|\tilde{f}(\cdot, 0)\|_{B S^{p}}<\infty .
$$

Consequently, the set $\Lambda:=\{\tilde{f}(\cdot, x): x \in B\}$ is bounded in $B S^{p}\left(\mathbb{R}, X_{\alpha-1}\right)$.

From Remark 4.1, for $\mu \in \mathcal{M}$, if we consider $u \in P A A\left(\mathbb{R}, X_{\beta}, \mu\right)$ and $f \in P A A S^{p} U\left(\mathbb{R} \times X_{\alpha-1}, X_{\beta}, \mu\right)$ satisfies (H3), then by Theorem 3.3, the function $h(\cdot):=\tilde{f}(\cdot, u(\cdot))$ belongs to $P A A S^{p}\left(\mathbb{R}, X_{\alpha-1}, \mu\right)$. Therefore, we have the following main result:

Theorem 4.4 Let $1 \leq p<\infty, 0 \leq \beta<\alpha \leq 1$ and $\mu \in \mathcal{M}$. Assume that (H1)-(H5) hold and $\tilde{f} \in$ $P A A S^{p} U\left(\mathbb{R} \times X_{\beta}, X_{\alpha-1}, \mu\right)$ satisfies (H6) with:

$$
\left\|L_{\tilde{f}}\right\|_{B S^{p}}<\left[m(\alpha, \beta)\left(\frac{q \gamma}{2}\right)^{(1-\alpha+\beta)}[q(\alpha-\beta-1) \Gamma(q(\alpha-\beta-1))]^{\frac{1}{q}}\left[\frac{1}{1-e^{-\frac{p \gamma}{2}}}\right]^{\frac{1}{p}}+c(\alpha, \beta)\left(\frac{2}{q \delta}\right)^{\frac{1}{q}}\left[\frac{1}{1-e^{-\frac{p \delta}{2}}}\right]^{\frac{1}{p}}\right]^{-1} .
$$

Then, equation (4.1) has a unique mild solution $u \in P A A\left(\mathbb{R}, X_{\beta}, \mu\right)$.

Proof. Consider the mapping $F: P A A\left(\mathbb{R}, X_{\beta}, \mu\right) \longrightarrow P A A\left(\mathbb{R}, X_{\beta}, \mu\right)$ defined by

$$
\begin{aligned}
(F u)(t) & :=\int_{-\infty}^{t} U_{\alpha-1}(t, s) P_{\alpha-1}(s) h(s) d s-\int_{t}^{+\infty} \tilde{U}_{\alpha-1}(t, s) Q_{\alpha-1}(t) h(s) d s \\
& =\left(F^{s} u\right)(t)+\left(F^{u} u\right)(t) \quad \text { for all } t \in \mathbb{R},
\end{aligned}
$$

where

$\left(F^{s} u\right)(t):=\int_{-\infty}^{t} U_{\alpha-1}(t, s) P_{\alpha-1}(s) \tilde{f}(s, u(s)) d s \quad$ and $\quad\left(F^{u} u\right)(t):=-\int_{t}^{+\infty} \tilde{U}_{\alpha-1}(t, s) Q_{\alpha-1}(t) \tilde{f}(s, u(s)) d s, t \in \mathbb{R}$.

It is clear that, $F\left(P A A\left(\mathbb{R}, X_{\beta}, \mu\right)\right) \subset P A A\left(\mathbb{R}, X_{\beta}, \mu\right)$. Moreover, we have

$$
\begin{aligned}
\left\|\left(F^{s} u\right)(t)-\left(F^{s} v\right)(t)\right\|_{\beta} & \leq \int_{-\infty}^{t}\left\|U_{\alpha-1}(t, s) P_{\alpha-1}(s)[\tilde{f}(s, u(s))-\tilde{f}(s, v(s))]\right\|_{\beta} d s \\
& \leq m(\alpha, \beta) \int_{-\infty}^{t} e^{-\gamma(t-s)}(t-s)^{\alpha-\beta-1}\|\tilde{f}(s, u(s))-\tilde{f}(s, v(s))\|_{\alpha-1} d s \\
& \leq m(\alpha, \beta)\left[\int_{-\infty}^{t} e^{-\frac{q \gamma(t-s)}{2}}(t-s)^{q(\alpha-\beta-1)} d s\right]^{\frac{1}{q}}\left[\int_{-\infty}^{t} e^{-\frac{p \gamma(t-s)}{2}}\|\tilde{f}(s, u(s))-\tilde{f}(s, v(s))\|_{\alpha-1}^{p} d s\right]^{\frac{1}{p}} \\
& =m(\alpha, \beta)\left(\frac{q \gamma}{2}\right)^{(1-\alpha+\beta)}[q(\alpha-\beta-1) \Gamma(q(\alpha-\beta-1))]^{\frac{1}{q}}\left[\int_{-\infty}^{t} e^{-\frac{p \gamma(t-s)}{2}}\|\tilde{f}(s, u(s))-\tilde{f}(s, v(s))\|_{\alpha-1}^{p} d s\right]^{\frac{1}{p}} \\
& =m(\alpha, \beta)\left(\frac{q \gamma}{2}\right)^{(1-\alpha+\beta)}[q(\alpha-\beta-1) \Gamma(q(\alpha-\beta-1))]^{\frac{1}{q}}\left[\sum_{k=1}^{\infty} \int_{t-k}^{t-k+1} e^{-\frac{p \gamma(t-s)}{2}} L^{p}(s) d s\right]^{\frac{1}{p}}\|u-v\|_{\infty} \\
& \leq\left\|L_{\tilde{f}}\right\|_{B S^{p}} m(\alpha, \beta)\left(\frac{q \gamma}{2}\right)^{(1-\alpha+\beta)}[q(\alpha-\beta-1) \Gamma(q(\alpha-\beta-1))]^{\frac{1}{q}}\left[\frac{1}{1-e^{-\frac{p \gamma}{2}}}\right]^{\frac{1}{p}}\|u-v\|_{\infty} .
\end{aligned}
$$


Arguing as above, we have also

$$
\begin{aligned}
\left\|\left(F^{u} u\right)(t)-\left(F^{u} v\right)(t)\right\|_{\beta} & \leq \int_{t}^{+\infty}\left\|\tilde{U}_{\alpha-1}(t-s) Q_{\alpha-1}(t)[\tilde{f}(s, u(s))-\tilde{f}(s, v(s))]\right\|_{\beta} d s \\
& \leq c(\alpha, \beta) \int_{t}^{+\infty} e^{\delta(t-s)}\|\tilde{f}(s, u(s))-\tilde{f}(s, v(s))\|_{\alpha-1} d s \\
& \leq c(\alpha, \beta)\left[\int_{t}^{+\infty} e^{\frac{q \delta(t-s)}{2}} d s\right]^{\frac{1}{q}}\left[\int_{t}^{+\infty} e^{\frac{p \delta(t-s)}{2}}\|\tilde{f}(s, u(s))-\tilde{f}(s, v(s))\|_{\alpha-1}^{p} d s\right]^{\frac{1}{p}} \\
& \leq\left\|L_{\tilde{f}}\right\|_{B S p} c(\alpha, \beta)\left(\frac{2}{q \delta}\right)^{\frac{1}{q}}\left[\frac{1}{1-e^{-\frac{p \delta}{2}}}\right]^{\frac{1}{p}}\|u-v\|_{\infty} .
\end{aligned}
$$

Consequently, we have

$\|F u-F v\|_{\infty} \leq\left\|L_{\tilde{f}}\right\|_{B S}\left[m(\alpha, \beta)\left(\frac{q \gamma}{2}\right)^{(1-\alpha+\beta)}[q(\alpha-\beta-1) \Gamma(q(\alpha-\beta-1))]^{\frac{1}{q}}\left[\frac{1}{1-e^{-\frac{p \gamma}{2}}}\right]^{\frac{1}{p}}+c(\alpha, \beta)\left(\frac{2}{q \delta}\right)^{\frac{1}{q}}\left[\frac{1}{1-e^{-\frac{p \delta}{2}}}\right]^{\frac{1}{p}}\right]\|u-v\|_{\infty}$.

Thus, $F$ has a unique fixed point belongs to $P A A\left(\mathbb{R}, X_{\beta}, \mu\right)$. This proves the result.

\section{$5 \quad \mu$-pseudo almost automorphic solutions for equation (1.1)}

Consider now the nonautonomous semilinear boundary differential equation:

$$
\left\{\begin{aligned}
u^{\prime}(t) & =A_{m}(t) u(t)+f(t, u(t)) \quad \text { for } t \in \mathbb{R}, \\
B(t) u(t) & =g(t, u(t)) \quad \text { for } t \in \mathbb{R},
\end{aligned}\right.
$$

where $\left(A_{m}(t), D\left(A_{m}(t)\right)\right)_{t \in \mathbb{R}}$ are linear operators on a Banach space $X, B(t): D\left(A_{m}(t)\right) \longrightarrow \partial X$, is a linear boundary operator and $f: \mathbb{R} \times X_{\beta} \longrightarrow X$ and $g: \mathbb{R} \times X_{\beta}^{t} \longrightarrow \partial X$ are locally integrable with resoect to first variable and Lipschitzian in the seconde one. We use the following assumptions:

(A1) There are Banach spaces $Z$ which are continuously embedded in $X$ and $\partial X$ respectively such that $A_{m}(t) \in \mathcal{L}(Z, X)$ and $B(t) \in \mathcal{L}(Z, \partial X)$ with: $\sup _{t \in \mathbb{R}}\left\|A_{m}(t)\right\|_{\mathcal{L}(Z, X)}$, $\sup _{t \in \mathbb{R}}\|B(t)\|_{\mathcal{L}(Z, \partial X)}<\infty$.

(A2) The restriction operators $A(t):=A_{m}(t) \mid \operatorname{ker}(B(t))$ for $t \in \mathbb{R}$, satisfy the conditions (2.1) and (2.2) with constants $\omega, \theta, K, L, \mu, v$. Hence, there is an evolution family $(U(t, s))_{s \leq t}$ on $X$ solving the equation (1.3) for $f=g=0$. Moreover, denote by $X_{m}^{t}:=\left(D(A(t)),\|\cdot\|_{A(t)}\right)$ where $\|\cdot\|_{A(t)}$ is the graph norm of $A(t)$. Then, $\|\cdot\|_{A(t)}$ and the norm of $Z$ are equivalent with constants being uniform in $t \in \mathbb{R}$.

(A3) The operator $B(t): Z \longrightarrow \partial X$ is surjective, i.e. $i m(B(t))=\partial X$ for each $t \in \mathbb{R}$.

(A4) For some $0<\tilde{\alpha}<1, Z$ is continuously embedded in $X_{\tilde{\alpha}}^{t}$ for each $t \in \mathbb{R}$ with uniformly bounded embedding constants and sup $\|D(t)\|_{\mathcal{L}(Z, X)}<\infty$.

where $D(t):=\left(B(t) \mid k e r\left(\omega-A_{m}(t)\right)\right)^{-1}, t \in \mathbb{R}$ are the Dirichlet maps given in [20, Lemma 1.2].

Note that the above assumptions are introduced the first time by G. Greiner in [20] in the autonomous case. By taking $\tilde{f}(t, x):=f(t, x)+\left(\omega-A_{\alpha-1}(t)\right) D(t) g(t, x)$, it follows from [5, Section 5], that equation (1.1) is equivalent to (4.1). Hence, a mild solution of equation (1.1) is a continuous function $u: \mathbb{R} \longrightarrow X_{\beta}$ satisfying the following variation of constants formula:

$$
u(t)=U(t, \sigma) u(\sigma)+\int_{\sigma}^{t} U_{\alpha-1}(t, s)\left[f(s, u(s))+\left(\omega-A_{\alpha-1}(s)\right) D(s) g(s, u(s))\right] d s \quad \text { for all } t \geq \sigma .
$$

Lemma 5.1 Let $1 \leq p<\infty$. Assume that (A1)-(A4) without the condition (2.2). Let $A_{m}(\cdot) \in A A S^{p}(\mathbb{R}, \mathcal{L}(Z, X))$ and $B(\cdot) \in A A S^{p}(\mathbb{R}, \mathcal{L}(Z, \partial X))$. Then, the following statements hold:

(i) $D(\cdot) \in A A S^{p}(\mathbb{R}, \mathcal{L}(Z, \partial X))$.

(ii) $R(\omega, A(\cdot)) \in A A S^{p}(\mathbb{R}, \mathcal{L}(Z, X))$.

(iii) $\left(\omega-A_{\alpha-1}(\cdot)\right) D(\cdot) \hat{g} \in A A S^{p}\left(\mathbb{R}, X_{\alpha-1}\right)$ for every $\hat{g} \in A A S^{p}(\mathbb{R}, \partial X)$ and $0 \leq \alpha<\tilde{\alpha}<1$. 
Proof. Let $\left(\sigma_{n}\right)_{n \geq 0}$ be a sequence of real numbers, since $A_{m}(\cdot) \in A A S^{p}(\mathbb{R}, \mathcal{L}(Z, X))$ and $B(\cdot) \in A A S^{p}(\mathbb{R}, \mathcal{L}(Z, \partial X))$. Then, there exists a subsequence $\left(s_{n}\right)_{n \geq 0}$ such that:

$$
\left(\int_{t}^{t+1}\left\|A_{m}\left(\sigma+s_{l}-s_{k}\right)-A_{m}(\sigma)\right\|^{p} d s\right)^{\frac{1}{p}} \rightarrow 0 \text { as } k, l \rightarrow \infty
$$

for all $t \in \mathbb{R}$ and

$$
\left(\int_{t}^{t+1}\left\|B\left(\sigma+s_{l}-s_{k}\right)-B(\sigma)\right\|^{p} d s\right)^{\frac{1}{p}} \rightarrow 0 \text { as } k, l \rightarrow \infty
$$

for all $t \in \mathbb{R}$.

(i) Let $y \in \partial X$ and $t \in \mathbb{R}$. By definition of $D(t)$, it follows for $s \in[t, t+1]$ that:

$$
\begin{array}{r}
\left(\omega-A_{m}(s)\right)\left(D\left(s+s_{l}-s_{k}\right) y-D(s) y\right)=\left(A_{m}\left(s+s_{l}-s_{k}\right)-A_{m}(s)\right) D\left(s+s_{l}-s_{k}\right) y=: \phi(s), \\
B(t)\left(D\left(s+s_{l}-s_{k}\right) y-D(s) y\right)=-\left(B\left(s+s_{l}-s_{k}\right)-B(s)\right) D\left(s+s_{l}-s_{k}\right) y=: \psi(s) .
\end{array}
$$

Hence

$$
D\left(s+s_{l}-s_{k}\right) y-D(s) y=R(\omega, A(s)) \phi(s)+D(s) \psi(s) .
$$

By assumptions, it holds that

$$
\begin{aligned}
\left(\int_{t}^{t+1}\left\|D\left(s+s_{l}-s_{k}\right) y-D(s) y\right\|_{Z}^{p}\right)^{\frac{1}{p}}= & \left(\int_{t}^{t+1}\|R(\omega, A(s)) \phi(s)+D(s) \psi(s)\|^{p}\right)^{\frac{1}{p}} \\
\leq & C\left[\left\|\phi^{b}(t)\right\|_{L^{p}([0,1], X)}+\left\|\psi^{b}(t)\right\|_{L^{p}([0,1], \partial X)}\right] \\
\leq & C\left[\left(\int_{t}^{t+1}\left\|\left(A_{m}\left(s+s_{l}-s_{k}\right)-A_{m}(s)\right)\right\|_{\mathcal{L}(Z, X)}^{p}\right)^{\frac{1}{p}}\right. \\
& \left.+\left(\int_{t}^{t+1}\left\|\left(B\left(s+s_{l}-s_{k}\right)-B(s)\right)\right\|_{\mathcal{L}(Z, \partial X)}^{p}\right)^{\frac{1}{p}}\right]\|y\|_{\partial X} .
\end{aligned}
$$

Therefore, from the limits in (5.2) and (5.3), we obtain that:

$$
\left(\int_{t}^{t+1}\left\|D\left(s+s_{l}-s_{k}\right)-D(s)\right\|_{\mathcal{L}(Z, \partial X)}^{p}\right)^{\frac{1}{p}} \rightarrow 0 \text { as } k, l \rightarrow \infty
$$

for all $t \in \mathbb{R}$.

(ii) Let $x \in X$ and $t \in \mathbb{R}$. For $s \in[t, t+1]$, take $y=R\left(\omega, A\left(s+s_{l}-s_{k}\right)\right) x-R(\omega, A(s)) x$, Then:

$$
\begin{array}{r}
\left(\omega-A_{m}(s)\right) y=\left(A_{m}\left(s+s_{l}-s_{k}\right)-A_{m}(s)\right) R\left(\omega, A\left(s+s_{l}-s_{k}\right)\right) x=: \tilde{\phi}(s), \\
B(t) y=-\left(B\left(s+s_{l}-s_{k}\right)-B(s)\right) R\left(\omega, A\left(s+s_{l}-s_{k}\right)\right) x=: \tilde{\psi}(s) .
\end{array}
$$

Hence,

$$
y=R(\omega, A(s)) \tilde{\phi}(s)+D(s) \tilde{\psi}(s)
$$

Therefore, arguing as in (i), we obtain that:

$$
\left(\int_{t}^{t+1}\left\|R\left(\omega, A\left(s+s_{l}-s_{k}\right)\right)-R(\omega, A(s))\right\|_{\mathcal{L}(Z, X)}^{p}\right)^{\frac{1}{p}} \rightarrow 0 \text { as } k, l \rightarrow \infty
$$

for all $t \in \mathbb{R}$.

(iii) To prove this statement, we need to introduce the following spaces:

For $1 \leq p<\infty$ and $0<\alpha<1$, we set $E(\mathbb{R})=B S^{p}(\mathbb{R}, X)$ and we define:

$$
A A S_{\alpha-1}^{p}(\mathbb{R}):=\left\{f \in E_{\alpha-1}(\mathbb{R}) \text { there exist }\left(f_{n}\right)_{n} \subset A A S^{p}(\mathbb{R}, X) \text { converging to } f \text { in } E_{\alpha-1}(\mathbb{R})\right\},
$$


where the extrapolated spaces $E_{\alpha-1}(\mathbb{R})$ are defined as in [5, Section 2], with the associated norms:

$$
\|f\|_{\alpha-1}:=\sup _{r>0} \sup _{t \in \mathbb{R}}\left(\int_{t}^{t+1} \| r^{\alpha} R\left(r,\left(\omega-A_{\alpha-1}(s)\right) f(s) \|^{p} d s\right)^{\frac{1}{p}}\right.
$$

and $E_{0}(\mathbb{R})=E(\mathbb{R}), E_{1}(\mathbb{R})=D(A(\cdot))$.

Under the assumptions (2.1), (H3) and (H4) it holds that

$$
E_{\alpha-1}(\mathbb{R}) \cong B S^{p}\left(\mathbb{R}, X_{\alpha-1}\right) \text { and } A A S_{\alpha-1}^{p}(\mathbb{R}) \cong A A S^{p}\left(\mathbb{R}, X_{\alpha-1}\right) .
$$

Furthermore, from (i) and (ii) and since $Z \hookrightarrow X$, the functions $D(\cdot) h$ and $f_{n}:=n R(n, A(\cdot)) D(\cdot) \hat{g} \in$ $A A S^{p}(\mathbb{R}, X)$ for $n>\omega$. Then, $A(\cdot) f_{n}=\left(n^{2} R(n, A(\cdot))-n\right) D(\cdot) \hat{g} \in A A S^{p}(\mathbb{R}, X)$. Moreover, by (2.1) and (A3), we claim that $f_{n}$ is uniformly bounded in the norm of $E_{\leq}(\mathbb{R})$. Since $f_{n} \rightarrow D(\cdot) \hat{g}$ in $B S^{p}(\mathbb{R}, X)$, we conclude by interpolation that $f_{n} \rightarrow D(\cdot) \hat{g}$ in $E_{\alpha}(\mathbb{R})$. As a consequence, $(\omega-A(\cdot)) f_{n} \rightarrow\left(\omega-A_{\alpha-1}(\cdot)\right) D(\cdot) \hat{g}$ in $E_{\alpha-1}(\mathbb{R})$. Hence, the result follows from the definition of $A A S_{\alpha-1}^{p}(\mathbb{R})$.

Lemma 5.2 Let $1 \leq p<\infty$ and $\mu \in \mathcal{M}$. Assume that (H5) and (A1)-(A4) without the condition (2.2). Let $A_{m}(\cdot) \in A A S^{p}(\mathbb{R}, \mathcal{L}(Z, X)), B(\cdot) \in A A S^{p}(\mathbb{R}, \mathcal{L}(Z, \partial X))$ and the functions $\hat{f} \in \operatorname{PAAS}^{p}(\mathbb{R}, X, \mu)$, $\hat{g} \in$ $\operatorname{PAAS}^{p}(\mathbb{R}, \partial X, \mu)$. Then, $h:=\hat{f}+\left(\omega-A_{\alpha-1}(\cdot)\right) D(\cdot) \hat{g} \in P A A S^{p}\left(\mathbb{R}, X_{\alpha-1}, \mu\right)$ for every $0 \leq \alpha<\tilde{\alpha}<1$.

Proof. Let $\hat{f}=\hat{f}_{0}+\xi \in P A A S^{p}(\mathbb{R}, X, \mu)$, where $\hat{f}_{0} \in A A S^{p}(\mathbb{R}, X)$ and $\xi \in \mathcal{E}^{p}(\mathbb{R}, X, \mu)$ and let $\hat{g}=$ $\hat{g}_{0}+\varphi \in P A A S^{p}(\mathbb{R}, \partial X, \mu)$, where $\hat{g} \in A A S^{p}(\mathbb{R}, \partial X)$ and $\varphi \in \mathcal{E}^{p}(\mathbb{R}, \partial X, \mu)$. Hence

$$
h=\underbrace{\hat{f}_{0}+\left(\omega-A_{\alpha-1}(\cdot)\right) D(\cdot) \hat{g}_{0}}_{h_{0}}+\underbrace{\xi+\left(\omega-A_{\alpha-1}(\cdot)\right) D(\cdot) \varphi}_{\psi} .
$$

Since $X \hookrightarrow X_{\alpha-1}, \hat{f}_{0} \in A A S^{p}(\mathbb{R}, X)$ and $\hat{g}_{0} \in A A S^{p}(\mathbb{R}, \partial X)$, it follows in view of Lemma 5.1 that $h_{0} \in$ $A A S^{p}\left(\mathbb{R}, X_{\alpha-1}\right)$ for every $0 \leq \alpha<\tilde{\alpha}<1$. It suffices to prove that $\psi \in \mathcal{E}^{p}\left(\mathbb{R}, X_{\alpha-1}, \mu\right)$ for every $0 \leq \alpha<$ $\tilde{\alpha}<1$. Let $r>0$, then we have

$$
\begin{aligned}
\frac{1}{\mu([-r, r])} \int_{[-r, r]}\left(\int_{t}^{t+1}\|\psi(s)\|_{\alpha-1}^{p} d s\right)^{\frac{1}{p}} d \mu(t) & \leq \frac{1}{\mu([-r, r])} \int_{[-r, r]}\left(\int_{t}^{t+1}\|\xi(s)\|_{\alpha-1}^{p} d s\right)^{\frac{1}{p}} d \mu(t) \\
& +\frac{M}{\mu([-r, r])} \int_{[-r, r]}\left(\int_{t}^{t+1}\|\phi(s)\|_{\partial X}^{p} d s\right)^{\frac{1}{p}} d \mu(t) \rightarrow 0 \text { as } r \rightarrow \infty,
\end{aligned}
$$

where $M:=\sup _{t \in \mathbb{R}}\left\|\left(\omega-A_{\alpha-1}(t)\right) D(t)\right\|_{\mathcal{L}\left(\partial X, X_{\alpha-1}\right)}<\infty$.

Theorems 4.2, 4.3 and Lemmas 5.1, 5.2 imply the following main result of this section on the existence of a $\mu$-pseudo almost automorphic mild solution to equation (1.1).

Theorem 5.1 Let $1 \leq p<\infty, 0 \leq \beta<\alpha \leq 1$ and $\mu \in \mathcal{M}$. Assume that $A_{m}(\cdot) \in \operatorname{AAS}^{p}(\mathbb{R}, \mathcal{L}(Z, X))$, $B(\cdot) \in A A S^{p}(\mathbb{R}, \mathcal{L}(Z, \partial X))$ and $f \in P A A S^{p} U\left(\mathbb{R} \times X_{\beta}, X, \mu\right), g \in P A A S^{p} U\left(\mathbb{R} \times X_{\beta}, \partial X, \mu\right)$ satisfy (H6) with small constants $\left\|L_{f}\right\|_{B S^{p}}$ and $\left\|L_{g}\right\|_{B S^{p}}$. Furthermore, if the hypotheses (A1)-(A4) and (H2), (H4)-(H5) hold. Then, equation (1.1) has a unique mild solution $u \in P A A\left(\mathbb{R}, X_{\beta}, \mu\right)$ given by:

$$
u(t)=\int_{\mathbb{R}} \Gamma_{\alpha-1}(t, s)\left[f(s, u(s))+\left(\omega-A_{\alpha-1}(s)\right) D(s) g(s, u(s))\right] d s \quad \text { for all } t \in \mathbb{R} .
$$

Proof. Define $\tilde{f}(t, x)=f(t, x)+\left(\omega-A_{\alpha-1}(\cdot)\right) D(\cdot) g(t, x)$ where $f \in P A P S^{p} U\left(\mathbb{R} \times X_{\beta}, X, \mu\right)$ and $g \in$ $P A A S^{p} U\left(\mathbb{R} \times X_{\beta}, \partial X, \mu\right)$. Since $M=\sup _{t \in \mathbb{R}}\left\|\left(\omega-A_{\alpha-1}(t)\right) D(t)\right\|_{\mathcal{L}\left(\partial X, X_{\alpha-1}\right)}<\infty$ and $Z \hookrightarrow X \hookrightarrow X_{\alpha-1}$, it follows from Lemma 5.2 that $\tilde{f} \in P A A S^{p} U\left(\mathbb{R} \times X_{\beta}, X_{\alpha-1}, \mu\right)$. Furthermore, the function $\tilde{f}$ satisfies (H6) with constant of Lipschitz $L_{\tilde{f}} \leq L_{f}+M L_{g}$. Hence, by a particular choice of $L_{f}$ and $L_{g}$ we can obtain $\left\|L_{\tilde{f}}\right\|_{B S^{p}}$ small enough. Hence, by Theorem 4.4, we deduce that there exists a unique mild solution $u \in P A A\left(\mathbb{R}, X_{\beta}, \mu\right)$ of equation of (1.1) satisfying the formula (5.4). 


\section{Application: Diffusion equation with inhomogeneous boundary con- ditions}

Let $\mu$ be a measure with a Radon-Nikodym derivative $\rho$ defined by:

$$
\rho(t)=\left\{\begin{array}{r}
e^{t} \text { for } t \leq 0 \\
1 \text { for } t>0 .
\end{array}\right.
$$

From [11, Example 3.6.], $\mu$ satisfies the hypothesis (H5).

In this section, we study the following diffusion equation with inhomogeneous boundary conditions:

$$
\left\{\begin{aligned}
\frac{\partial u(t, x)}{\partial t} & =\frac{\partial^{2} u(t, x)}{\partial x^{2}}+b(t) u(t, x), \quad(t, x) \in \mathbb{R} \times(0, \pi), \\
c(t, x) \frac{\partial u(t, x)}{\partial n} & =\Phi(t, u(t, x)), \quad(t, x) \in \mathbb{R} \times \Gamma
\end{aligned}\right.
$$

where $\Gamma=\{0\} \cup\{\pi\}, b \in L^{\infty}(\mathbb{R}), c \in B S^{1}\left(\mathbb{R}, C^{1}(\Gamma)\right) \cap L^{\infty}\left(\mathbb{R}, C^{1}(\Gamma)\right)$ are given. The function $g: \mathbb{R} \times X \longrightarrow$ $\partial X$ defined by

$$
g(t, \varphi)(x)=\Phi(t, \varphi(x))=\frac{a(t)}{1+|\varphi(x)|},
$$

where $a(t):=a_{1}(t)+\left(\arctan (t)-\frac{\pi}{2}\right)$, with

$$
a_{1}(t)=\sum_{n \geq 1} \beta_{n}(t)
$$

such that, for every $n \geq 1$

$$
\beta_{n}(t)=\sum_{i \in P_{n}} H\left(n^{2}(t-i)\right),
$$

with $P_{n}=3^{n}(2 \mathbb{Z}+1)$ and $H \in C_{0}^{\infty}(\mathbb{R}, \mathbb{R})$ with support in $\left(\frac{-1}{2}, \frac{1}{2}\right)$ such that

$$
H \geq 0, \quad H(0)=1 \quad \text { and } \quad \int_{\frac{-1}{2}}^{\frac{1}{2}} H(s) d s=1 .
$$

By the proof in [11, Section 5.], $t \longmapsto \arctan (t)-\frac{\pi}{2}$ belongs to $\mathcal{E}(\mathbb{R}, \mathbb{R}, \mu)$. Otherwise, from [17] and references therein, $a_{1} \in C^{\infty}(\mathbb{R}, \mathbb{R})$, but $a_{1} \notin A A(\mathbb{R}, \mathbb{R})$ since it is not bounded. However, $a_{1} \in A A S^{1}(\mathbb{R}, \mathbb{R})$.

In order to rewrite equation (1.1) as an abstract differential equation, we take the spaces $X:=L^{2}((0, \pi), \mathbb{R})$, $Z:=H^{2}((0, \pi), \mathbb{R})$ and $\partial X:=H^{\frac{1}{2}}((0, \pi), \mathbb{R})$ equipped with there usual norms. Moreover, we consider the following statement:

$$
\left\{\begin{array}{rlrl}
A_{m}(t) & =\frac{\partial^{2}}{\partial x^{2}}+b(t):=A_{m}+b(t) & \text { for } t \in \mathbb{R}, \\
D\left(A_{m}(t)\right) & =D\left(A_{m}\right)=H^{2}((0, \pi), \mathbb{R}) & & \text { for } t \in \mathbb{R},
\end{array}\right.
$$

where $b(t):=b(t, \cdot) \in A A S^{1}(\mathbb{R}, X)$ and the boundary operator

$$
\begin{aligned}
\left.B(t): H^{2}(0, \pi), \mathbb{R}\right) & \longrightarrow H^{\frac{1}{2}}(\Gamma, \mathbb{R}) \\
v & \mapsto\left(c(t) \frac{\partial v}{\partial n}\right) \mid \Gamma
\end{aligned}
$$

Hence, we define $A(t):=A_{m}(t) \mid \operatorname{ker}(B(t))$ where $\operatorname{ker}(B(t))=H^{2}((0, \pi), \mathbb{R}) \cap H_{0}^{1}((0, \pi), \mathbb{R})$. Since $c \in$ $L^{\infty}(\mathbb{R} \times \Gamma)$, it follows in view of the Trace Theorem [22, Theorem 9.4] that $B(t)$ is surjective and bounded uniformly in $t \in \mathbb{R}$. Moeover, $X_{\alpha}^{t}=H^{2 \alpha}((0, \pi), \mathbb{R})$ with uniformly equivalent norms, for $\alpha \in\left(\frac{1}{3}, \frac{1}{2}\right)$ and that the Dirichlet map $D(t): Z \longrightarrow \partial X$ is uniformly bounded for $t \in \mathbb{R}$, see [4, Example IV.2.6.3 ]. It 
is well known that the graph norm of $A(t)$ is uniformly equivalent the norm of $Z$ and that the operator $A:=A_{m} \mid \operatorname{ker}(B(t))$ is a generator of an analytic semigroup $(T(t))_{t \geq 0}$ on $X$. Moreover, we have

$$
T(t) f=\sum_{n=0}^{\infty} e^{-n^{2} \pi^{2} t}<f, e_{n}>_{L^{2}[0, \pi]} e_{n} \quad \text { for all } t \in \mathbb{R}, f \in X,
$$

where $\left\{e_{n} ; n \geq 0\right\}$ is the family of eigenvectors associated to the egeinvalues $\left\{-n^{2} \pi^{2} ; n \geq 0\right\}$. Take $v(t)(\cdot):=u(t, \cdot)$, hence the equation (1.1) has the following abstract form:

$$
\left\{\begin{aligned}
v^{\prime}(t) & =A_{m}(t) v(t) \quad \text { for } t \in \mathbb{R}, \\
B(t) v(t) & =g(t, v(t)) \quad \text { for } t \in \mathbb{R} .
\end{aligned}\right.
$$

The operators $(A(t))_{t \in \mathbb{R}}$ generate an evolution family $(U(t, s))_{t \geq s}$ on $X$ given by:

$$
U(t, s) f=\sum_{n=0}^{\infty} e^{\int_{s}^{t}\left[b(\tau)-n^{2} \pi^{2}\right] d \tau}<f, e_{n}>_{L^{2}[0, \pi]} e_{n} \quad \text { for all } t \geq s, f \in X,
$$

which satisfies (2.1) and (2.2). Furthermore, we have

$$
\begin{aligned}
& \|U(t, s) f\|^{2}=<U(t, s) f, U(t, s) f>_{L^{2}[0, \pi]} \\
& =\sum_{n=0}^{\infty} \sum_{m=0}^{\infty} e^{\int_{s}^{t}\left[b(\tau)-n^{2} \pi^{2}\right] d \tau} e^{\int_{s}^{t}\left[b(\tau)-m^{2} \pi^{2}\right] d \tau}<f, e_{n}>_{L^{2}[0, \pi]}<f, e_{m}>_{L^{2}[0, \pi]}<e_{n}, e_{m}>_{L^{2}[0, \pi]} \\
& \leq \sum_{n=0}^{\infty} e^{2 \int_{s}^{t}\left[b(\tau)-n^{2} \pi^{2}\right] d \tau}\|f\|^{2} \\
& =\left(\sum_{n=0}^{\infty} e^{\int_{s}^{t}\left[b(\tau)-n^{2} \pi^{2}\right] d \tau}\|f\|\right)^{2} \quad \text { for all } t \geq s, f \in X .
\end{aligned}
$$

Hence,

$$
\|U(t, s) f\|=\sum_{n=0}^{\infty} e^{\int_{s}^{t}\left[b(\tau)-n^{2} \pi^{2}\right] d \tau}\|f\| \quad \text { for all } t \geq s, f \in X
$$

Consequently, the hypotheses (A1)-(A4) and (H4) are satisfied.

Let us prove that $(U(t, s))_{t \geq s}$ has an exponential dichotomy on $\mathbb{R}$. Indeed, the series given in (6.3) is uniformly convergent which implies that the sequence $\left(e^{t}\left[b(\tau)-n^{2} \pi^{2}\right] d \tau\right)$ goes to 0 as $n \rightarrow 0$. Let $N \geq 1$ large enough, since $b \in A A S^{1}(\mathbb{R}, X)$, it follows that:

$$
\begin{aligned}
\sum_{n=0}^{\infty} e^{\int_{s}^{t}\left[b(\tau)-n^{2} \pi^{2}\right] d \tau} & \leq \sum_{n=0}^{\infty} e^{[t]+1} \int_{k=[s]}^{k+1} b(\tau) d \tau-n^{2} \pi^{2}(t-s) \\
& \leq M \sum_{n=0}^{\infty} e^{\left(|b|_{B S^{1}}-n^{2} \pi^{2}\right)(t-s)}, \quad \text { with } M=e^{2|b|_{B S^{1}}} \\
& =M \sum_{n=0}^{N} e^{\left(|b|_{B S^{1}}-n^{2} \pi^{2}\right)(t-s)}+M \sum_{n=N+1}^{\infty} e^{\left[|b|_{B S^{1}}-n^{2} \pi^{2}\right](t-s)}
\end{aligned}
$$

Therefore, there exist $\delta, \tilde{M}>0$ and the projections $P(t):=P$ and $Q(t):=Q=I-P$, for $t \in \mathbb{R}$ defined as $\operatorname{Rank}(Q)=N$ and $\operatorname{Rank}(P)=\infty$, since it can be seen as $Q=\operatorname{diag}(1, \ldots, 1,0,0, \ldots)$ with $N$ numbers 1 at the diagonal. Then, we have the following decomposition:

$$
\begin{cases}\|U(t, s) P(s)\| \leq \tilde{M} e^{-\delta(t-s)}, & t, s \in \mathbb{R}, s \leq t \\ \|U(s, t) Q(t)\| \leq \tilde{M} e^{-\delta(s-t)}, & t, s \in \mathbb{R}, s>t\end{cases}
$$

Lemma 6.1 The function $g$ satisfies the condition (H6) for $p=1$ and $L_{g}:=|m|_{\infty}|a(\cdot)| \in B S^{1}(\mathbb{R}, \mathbb{R})$. 
Proof. Let $\varphi_{1}, \varphi_{2} \in X$. Then,

$$
\begin{aligned}
\int_{[0, \pi]}\left|g\left(t, \varphi_{1}\right)(x)-g\left(t, \varphi_{2}\right)(x)\right|^{2} d x & =[a(t)]^{2} \int_{[0, \pi]}\left|\frac{1}{1+\left|\varphi_{1}(x)\right|}-\frac{1}{1+\left|\varphi_{2}(x)\right|}\right|^{2} d x \\
& \leq[a(t)]^{2} \int_{[0, \pi]}\left|\varphi_{1}(x)-\varphi_{2}(x)\right|^{2} d x \\
& \leq[a(t)]^{2}\left\|\varphi_{1}-\varphi_{2}\right\|^{2} \quad \text { for all } t \in \mathbb{R} .
\end{aligned}
$$

Hence

$$
\left\|g\left(t, \varphi_{1}\right)-g\left(t, \varphi_{1}\right)\right\| \leq|a(t)|\left\|\varphi_{1}-\varphi_{2}\right\| \quad \text { for all } t \in \mathbb{R} .
$$

The result follows from the fact that $a \in P A A S^{1}(\mathbb{R}, \mathbb{R}, \mu)$.

Lemma 6.2 Let $b \in A A S^{1}(\mathbb{R}, \mathbb{R})$ and $c \in A A S^{1}\left(\mathbb{R}, C^{1}(\Gamma)\right)$. Then, the following hold

(i) $A_{m}(t) \in \mathcal{L}(Z, X)$ with $\sup _{t \in \mathbb{R}}\left\|A_{m}(t)\right\|_{\mathcal{L}(Z, X)}<\infty$. Moreover, $A_{m}(\cdot) \in A A S^{1}(\mathbb{R}, \mathcal{L}(Z, X))$.

(ii) $B(t) \in \mathcal{L}(Z, \partial X)$ with $\sup _{t \in \mathbb{R}}\|B(t)\|_{\mathcal{L}(Z, \partial X)}<\infty$. Moreover, $B(\cdot) \in A A S^{p}(\mathbb{R}, \mathcal{L}(Z, \partial X))$.

Proof. Let $b \in A A S^{1}(\mathbb{R}, \mathbb{R}) \cap L^{\infty}(\mathbb{R})$ and $c \in A A S^{1}\left(\mathbb{R}, C^{1}(\Gamma)\right) \cap L^{\infty}\left(\mathbb{R}, C^{1}(\Gamma)\right)$.

(i) Let $\varphi \in X$. Since $Z \hookrightarrow X$, it holds that,

$$
\begin{aligned}
\left\|A_{m}(t) \varphi\right\| & \leq\left\|A_{m} \varphi\right\|+|b(t)|\|\varphi\| \\
& \leq\left\|A_{m}\right\|_{\mathcal{L}(Z, X)}\|\varphi\|_{Z}+C|b(t)|\|\varphi\|_{Z} \\
& =\left[\left\|A_{m}\right\|_{\mathcal{L}(Z, X)}+C|b(t)|\right]\|\varphi\|_{Z}, \quad t \in \mathbb{R} .
\end{aligned}
$$

Hence,

$$
\begin{aligned}
\left\|A_{m}(t)\right\|_{\mathcal{L}(Z, X)} & \leq\left[\left\|A_{m}\right\|_{\mathcal{L}(Z, X)}+C|b(t)|\right] \\
& \leq\left[\left\|A_{m}\right\|_{\mathcal{L}(Z, X)}+C|b|_{\infty}\right], \quad t \in \mathbb{R} .
\end{aligned}
$$

Then,

$$
\sup _{t \in \mathbb{R}}\left\|A_{m}(t)\right\|_{\mathcal{L}(Z, X)}<\infty .
$$

Now, we show that $A_{m}(\cdot) \in A A S^{1}(\mathbb{R}, \mathcal{L}(Z, X))$. Let $\left(\sigma_{l}\right)_{l \geq 0}$ be a sequence of real numbers, since $b \in$ $A A S^{1}(\mathbb{R}, \mathbb{R})$, there exists a subsequence $\left(s_{l}\right)_{l \geq 0}$ such that

$$
\int_{t}^{t+1}\left|b\left(s+s_{l}-s_{k}\right)-b(s)\right| d s \rightarrow 0 \text { as } k, l \rightarrow \infty .
$$

for all $t \in \mathbb{R}$.

Therefore, from (6.4), we obtain that

$$
\int_{t}^{t+1}\left\|A_{m}\left(s+s_{l}-s_{k}\right)-A_{m}(s)\right\|_{\mathcal{L}(Z, X)} d s \leq C \int_{t}^{t+1}\left|b\left(s+s_{l}-s_{k}\right)-b(s)\right| d s, \quad k, l \in \mathbb{N}, t \in \mathbb{R} .
$$

Then, by (6.5), we deduce the result.

(ii) We follow the proof as in (i).

Then, we have the following main result.

Theorem 6.1 Let $0 \leq \beta<\alpha \leq 1$. Assume that $b \in A A S^{1}(\mathbb{R}, \mathbb{R}) \cap L^{\infty}(\mathbb{R}), c \in \operatorname{AAS}^{1}\left(\mathbb{R}, C^{1}(\Gamma)\right) \cap$ $L^{\infty}\left(\mathbb{R}, C^{1}(\Gamma)\right)$. If we take $\left\|L_{g}\right\|_{B S^{1}}$ small enough. Then equation (6.2) has a unique mild solution $u \in$ $P A A\left(\mathbb{R}, X_{\beta}, \mu\right)$. 


\section{References}

[1] P. Acquistapace and B. Terreni, A unified approach to abstract linear nonautonomous parabolic equations, Rendiconti del Seminario Matematico della Università di Padova, 78, 47-107, (1987).

[2] P. Acquistapace, Evolution operators and strong solutions of abstract linear parabolic equations, Differential Integral Equations, 1, 433-457, (1988).

[3] A. N. Akdad, B. Es-sebbar and K. Ezzinbi, Composition Theorems of Stepanov $\mu$-Pseudo Almost Automorphic Functions and Applications to Nonautonomous Neutral Evolution Equations, Differential Equations and Dynamical Systems, 1-20, (2015).

[4] H. Amann, Linear and Quasilinear Parabolic Problems, Volume 1: Abstract Linear Theory, Birkhaüser, Basel, 1995.

[5] M. Baroun, L. Maniar and R. Schnaubelt, Almost Periodicity of Parabolic Evolution Equations with Inhomogeneous Boundary Values, Integral equations and operator theory, 65, 169-193, (2009).

[6] M. Baroun, K. Ezzinbi, K. Khalil and L. Maniar, Pseudo almosr periodic solutions for some parabolic evolution equations with Stepanov-like pseudo almost periodic forcing terms, Journal of Mathematical Analysis and Applications, 462, (1), 233-262, (2018).

[7] S. Bochner, Abstrakte fastperiodische funktionen, Acta Mathematica, 61, (1), 149-184, (1933).

[8] S. Bochner, A new approach to almost periodicity, Proceedings of the National Academy of Sciences of the United States of America, 48, (12), 2039-2043, (1962).

[9] H. Bohr. Zur theorie der fastperiodischen funktionen. Acta Mathematica, 46, (1-2), 101-214, (1925).

[10] M. Baroun, S. Boulite, G.M. N'Guérékata and L. Maniar, Almost automorphy of parabolic evolution equations, Electronic Journal of Differential Equations, 60, 1-9, (2008).

[11] J. Blot, P. Cieutat and K. Ezzinbi, Measure theory and pseudo almost automorphic functions: New developments and applications, Nonlinear Analysis, 75, 2426-2447, (2012).

[12] J. Blot, P. Cieutat and K. Ezzinbi, New approach for weighted pseudo almost periodic functions under the light of measure theory, basic results and applications, Applicable Analysis, 92, (3), 493-526, (2013).

[13] T. Diagana, Almost Automorphic Type and Almost Periodic Type Functions in Abstract Spaces, Springer International Publishing Switzerland, 2013.

[14] T. Diagana, Weighted pseudo almost periodic functions and applications. Comptes Rendus Mathématique, 343, (10), 643-646, (2006).

[15] T. Diagana and G.M. N'Guérékata, Almost automorphic solutions to some classes of partial evolution equations, Applied Mathematical Letters, 20, (4), 462-466, (2007).

[16] B. Es-sebbar, K. Ezzinbi, Almost periodicity and almost automorphy for some evolution equations using Favard's theory in uniformly convex Banach spaces, Semigroup Forum, (2016).

[17] B. Es-sebbar, K. Ezzinbi, Stepanov ergodic perturbations for some neutral partial functional differential equations, Mathematical Methods in the Applied Sciences, 39, (8), 1945-1963, (2016).

[18] R. Engel and K J. Nagel, One-Parameter Semigroups for Linear Evolution Equations, Vol.194, Springer-Verlag: New York, 2000.

[19] Z. Fan J. Liang and T. J. Xiao, Composition of Stepanov-like pseudo almost automorphic functions and applications to nonautonomous evolution equations, Nonlinear Analysis: Real World Applications, 13, 131-140, (2012).

[20] G. Greiner, Perturbing the boundary conditions of a generator, Houston Journal of Mathematics, 13, 213-229, (1987). 
[21] J. Hong, R. Obaya, and A. Sanz, Almost periodic type solutions of some differential equations with piecewise constant argument, Nonlinear Analysis: Theory Methods and Applications, 45, (6), 661-688, (2001).

[22] J. L. Lions, Problèmes aux limites non homogènes et applications, Dunod, Paris, 1968.

[23] A. Lunardi, Analytic Semigroups and Optimal Regularity in Parabolic Problems, Birkhauser, Basel, Boston, Berlin, 1995.

[24] L. Maniar and R. Schnaubelt, Almost periodicity of inhomogeneous parabolic evolution equations, Lecture Notes in Pure and Applied Mathematics, 234, Dekker, New York, 299-318, (2003).

[25] G.M. N'Guérékata, Topics in Almost Automorphy, Springer, New York, Boston, Dordrecht, London, Moscow, 2005.

[26] G. M. N'Guérékata and A. Pankov, Stepanov-like almost automorphic functions and monotone evolution equations, Nonlinear Analysis, 68, 2658-2667, (2008).

[27] A. Pazy, Semigroups of Linear Operators and Applications to Partial Differential Equations, SpringerVerlag, New York, 1983.

[28] L. Schwartz, Topologie Générale et Analyse Fonctionnelle, Hermann, Paris, (in French), 1976.

[29] W. Shen and Y. Yi. Almost automorphic and almost periodic dynamics in skew-product semiflows, Memoirs of the American Mathematical Society, 136, (647), 1998.

[30] W. Stepanov, Ueber einige verallgemeinerungen der fastperiodischen functionen. Annals of Mathematics, 95, 473-498, (1926).

[31] T. J. Xiao, J. Liang and J. Zhang, Pseudo almost automorphic solutions to semilinear differential equations in Banach spaces, Semigroup Forum, 76, (3), 518-524, (2008).

[32] C. Y. Zhang, Pseudo almost periodic solutions of some differential equations. Journal of Mathematical Analysis and Applications, 181, (1, 62-76 ), (1994).

[33] R. Zhang, Y.K. Chang and G.M. N'Guérékata, New composition theorems of Stepanov-like weighted pseudo almost automorphic functions and applications to nonautonomous evolution equations, Nonlinear Analysis: Real World Applications, 13, 2866-2879, (2012). 